University of Nebraska - Lincoln

DigitalCommons@University of Nebraska - Lincoln

3-16-2009

\title{
Analytic formulas for above-threshold ionization or detachment plateau spectra
}

\author{
M. V. Frolov \\ Voronezh State University, \\ N. L. Manakov \\ Voronezh State University,, manakov@phys.vsu.ru \\ Anthony F. Starace \\ University of Nebraska-Lincoln, astarace1@unl.edu
}

Follow this and additional works at: https://digitalcommons.unl.edu/physicsstarace

Part of the Physics Commons

Frolov, M. V.; Manakov, N. L.; and Starace, Anthony F., "Analytic formulas for above-threshold ionization or detachment plateau spectra" (2009). Anthony F. Starace Publications. 167.

https://digitalcommons.unl.edu/physicsstarace/167

This Article is brought to you for free and open access by the Research Papers in Physics and Astronomy at DigitalCommons@University of Nebraska - Lincoln. It has been accepted for inclusion in Anthony F. Starace Publications by an authorized administrator of DigitalCommons@University of Nebraska - Lincoln. 


\title{
Analytic formulas for above-threshold ionization or detachment plateau spectra
}

\author{
M. V. Frolov and N. L. Manakov \\ Department of Physics, Voronezh State University, Voronezh 394006, Russia \\ Anthony F. Starace \\ Department of Physics and Astronomy, The University of Nebraska, Lincoln, Nebraska 68588-0111, USA
}

(Received 9 January 2009; published 16 March 2009)

\begin{abstract}
Closed-form analytic formulas are derived in the tunneling limit for both above-threshold detachment (ATD) of negative ions and above-threshold ionization (ATI) of neutral atoms. These formulas are shown to give precise agreement with essentially exact single-active-electron numerical results for detached or ionized electron energies corresponding to the high-energy end of the ATD and ATI plateaus (with only a small constant shift of electron energies being required in the case of ATI). These formulas for ATI and ATD rates thus provide an analytic explanation for the well-known oscillatory patterns of ATI and ATD rates as functions of electron energy and of the parameters of the laser field. They also provide an analytic explanation for the dependence of these rates on the initial orbital angular momentum of the active electron. Most significantly, these formulas provide a fully quantum justification of the well-known classical three-step scenario for strong-field ionization and detachment spectra in the high-energy region of the ATI or ATD plateau.
\end{abstract}

DOI: 10.1103/PhysRevA.79.033406

PACS number(s): $32.80 . \mathrm{Rm}, 32.80 . \mathrm{Wr}$

\section{INTRODUCTION}

One of the most important phenomena in intense laseratom physics is above-threshold ionization (ATI), i.e., the ionization of an atom with absorption of a larger number $(n)$ of laser field photons than the minimum necessary for ionization. Besides its intrinsic interest as a source of highenergy electrons in laser-atom interactions, ATI plays a key role in other laser-atom phenomena, such as high-order harmonic generation. For this reason it has continued to be actively investigated now for more than two decades (see, e.g., Refs. [1,2]).

The energy spectra of ATI electrons may be divided into distinct regions depending on the relation between the ionized electron's energy $E$ and the ponderomotive energy, $u_{p}$ $=e^{2} F^{2} /\left(4 m \omega^{2}\right)$ [i.e., the average energy of free-electron oscillations in a laser electric field, $\mathbf{F}(t)=\mathbf{e}_{z} F \cos \omega t$, where $F$ and $\omega$ are the field amplitude and frequency]. The two most important spectral regions are the low-energy (or Keldysh) part and the high-energy (plateau or rescattering) part. The first one is well described by the Keldysh theory of tunneling ionization [3]. For low frequencies $\left(\hbar \omega \ll\left|E_{0}\right|\right.$, where $\left|E_{0}\right|$ is the energy of the bound electron) it extends in energy up to $2 u_{p}$ [which corresponds to the maximum instantaneous energy of free-electron oscillations in the field $\mathbf{F}(t)]$. The highenergy part extends up to $\sim 10 u_{p}$ and the magnitudes of the high-energy ATI rates are several orders of magnitude less than those in the Keldysh part. This difference in the magnitudes of ATI rates for these two regions originates from the fact that tunneling ionization requires only a minimal account of the electron-atom interaction (i.e., only on the level of the initial bound-state wave function), while the description of the high-energy ATI spectrum requires a more complete account of this interaction. The most spectacular feature of the high-energy ATI spectrum is that ATI electron peaks corresponding to absorption of different numbers of photons have nearly equal magnitude, producing thus the so-called ATI plateau. The interpretation of this plateau structure in the ATI process is given by the well-known three-step (rescattering) scenario [2], in which an electron (i) tunnels to the continuum under the influence of an oscillating laser field, (ii) is accelerated away from the atom by the laser field (acquiring a kinematic momentum along the direction of laser polarization), and (iii) after the field changes sign, is accelerated back to the atomic core, from which it scatters, changing the direction of its momentum and producing the observed angular distribution of ATI electrons.

Although plateau structures in ATI spectra are well established both experimentally and theoretically [by means of numerical solutions of the time-dependent Schrödinger equation (TDSE)], there does not yet exist a closed-form analytic formula describing such plateau structures that is similar to the Keldysh formula for tunneling ionization [3]. Semianalytical quantum analyses of the ATI process that confirm the rescattering scenario are based primarily on two alternative approaches: (i) the use of various modifications of the strong-field (or Keldysh) approximation, or (ii) the use of some exactly solvable quantum model for above-threshold detachment (ATD) of an electron bound in a short-range potential, i.e., neglecting the long-range (Coulomb) tail of the atomic core potential.

Different modifications of the strong-field approximation have been suggested [4-7]. All of them are based on a perturbativelike account of the atomic potential beyond the Keldysh approach. Additionally, estimations of the multidimensional (up to five) integrals for the ATI amplitudes that occur within these modifications employ either saddle-point methods [including quasiclassical analyses in terms of electron trajectories (cf. Ref. [2] for a review)] or more subtle methods for estimating integrals involving oscillating functions, such as the uniform approximation [8] (cf. also Ref. [9]).

Among the exactly solvable models is one (cf. Refs. $[10,11])$ that is based on the exact solution of the TDSE for an electron in both a zero-range potential and a strong laser field [12]. Another more general model $[13,14]$ is based on 
the time-dependent effective range (TDER) theory, within which the zero-range potential model is a special case (for bound $s$ states and an effective range parameter of zero). The great advantage of exactly solvable models is the possibility to check the accuracy of different approximate theories, by means of self-consistent comparison of approximate and exact results within the framework of the same approach. Although Coulomb effects are usually neglected in semianalytical analyses, results of such short-range potential models exhibit in many instances good qualitative agreement with numerical results for neutral atoms [2]. However, most such semianalytic ATI or ATD theories require in a final step the numerical evaluation of one or more complicated temporal integrals. A closed analytic formula for ATI angular distributions in the tunneling limit was obtained (as far as we are aware) only in Ref. [9]. However, its accuracy and conditions of applicability are not as yet established.

An important recent extension of the rescattering scenario for ATI consists of the ad hoc factorization of the angular distribution of ATI electrons near the ATI plateau cutoff as a product of the elastic-scattering cross section of the active electron from the positive ion (in the case of ATI) $[15,16]$ or the neutral atom (in the case of ATD) [17] and an "electron wave packet," $W(E)$, originating from the first two steps of the three-step ATI or ATD scenario (i.e., the tunneling ionization and subsequent laser acceleration steps). This parametrization has been the subject of recent experimental studies $[18,19]$. Although the results in Refs. [15-19] support this parametrization and show that the energy dependence of the electron wave packet $W(E)$ is largely independent of the target atom (based on both the experimental measurements and corresponding numerical solutions of the TDSE for a single active electron), the analytic structure of the function $W(E)$ remains a "black box." Hence an analytic justification for this parametrization as well as an explicit form for $W(E)$ is very desirable, even for an atomic system that may be regarded as a special case [owing to the demonstrated insensitivity of $W(E)$ to the particular system].

In this paper we derive simple analytic formulas (involving a single Airy function) for the amplitudes and electron angular distributions for ATD of an electron bound in a short-range potential. These formulas are shown to provide excellent agreement with exact numerical results of the TDER theory over the high-energy part of the ATD plateau (and beyond). Moreover, these results provide a quantum justification for the classical three-step rescattering scenario as well as a correction to the well-known classical $\left(\sim 10 u_{p}\right)$ law for the energy position of the ATI or ATD plateau cutoff. They also confirm the above-discussed two-term parametrization of the electron angular distribution and provide an explicit formula for $W(E)$ that shows its insensitivity to the orbital symmetry (i.e., the angular momentum) of the boundelectron wave function. Finally, these formulas provide clear explanations for various qualitative features of ATD spectra, such as: (i) the dependence of the oscillatory patterns of ATD rates in the plateau region on the electron momentum, (ii) the dependence of the oscillatory patterns of the $n$-photon ATD rate on the laser parameters, and (iii) the dependence of the ATD rates on the orbital angular momentum $l$ of the bound electron. The simplicity and transparent physical meaning of these analytic formulas for ATD rates allow one to generalize them to the case of neutral atoms. As we show, the resulting Coulomb-modified formulas give results that agree very well with results for the shapes of electron angular distributions in the high-energy region of the ATI plateau that are obtained numerically by solving the TDSE for the case of the hydrogen atom.

The paper is organized as follows. In Sec. II we give an alternate expression for the exact ATD amplitude in the TDER theory from that in Refs. $[13,14]$. This form of the exact ATD amplitude is then used in Sec. III to obtain an analytic formula for the ATD amplitude within the quasiclassical (tunneling) limit. In Sec. IV we discuss this analytic three-step result for the ATD amplitude and demonstrate (by comparison with exact TDER results) the accuracy of its description of the high-energy part of the ATD plateau. The generalization our analytic result for ATD rates to the case of neutral atoms is presented in Sec. V. Appendixes A-C provide mathematical derivations of some of the more detailed intermediate results needed to obtain the analytical formulas for the ATD amplitude and rate in Secs. II-IV.

\section{INTEGRAL REPRESENTATION FOR THE EXACT ATD AMPLITUDE IN TDER THEORY}

We consider a single active electron in a bound state, $\psi_{\kappa l m}(\mathbf{r})=R_{\kappa l}(r) Y_{l, m}(\hat{\mathbf{r}})$, of the potential $U(r)$, with angular momentum $l$ and energy $E_{0}=-(\hbar \kappa)^{2} /(2 m)$, that interacts with a monochromatic laser electric field $\mathbf{F}(t)$. Within the complex quasienergy (or Floquet) approach, the bound state $\psi_{\kappa l m}(\mathbf{r})$, when subjected to a monochromatic laser field, evolves to the quasistationary quasienergy state (QQES) $\Phi_{\epsilon}(\mathbf{r}, t)$. The complex quasienergy $\epsilon$ describes the position $(\operatorname{Re} \epsilon)$ and the total decay rate $(\Gamma=-2 \operatorname{Im} \epsilon / \hbar)$ of the laserdressed state $\psi_{\kappa l m}(\mathbf{r})$, while the asymptotic form of the periodic (in time) QQES wave function $\Phi_{\epsilon}(\mathbf{r}, t)$ at large $r$ provides an accurate $a b$ initio definition for the $n$-photon ATI (ATD) amplitude $\mathcal{A}_{n}\left(\mathbf{p}_{n}\right)$ in terms of the Fourier coefficients of $\Phi_{\epsilon}(\mathbf{r}, t)$. The momentum $p_{n}=\sqrt{2 m\left(E_{0}+n \hbar \omega-u_{p}\right)}$ is that of the ionized (detached) electron. For details, see, e.g., the reviews in $[20,21]$.

To simplify the solution of the four-dimensional (i.e., based on the TDSE) eigenvalue problem for obtaining $\Phi_{\epsilon}(\mathbf{r}, t)$ and $\epsilon$ for the case of a general potential $U(r)$, we assume $U(r)$ to be of short range (of radius $r \approx r_{c}$ ) and to support only a single weakly bound (negative-ion) state $\psi_{\kappa l m}(\mathbf{r})$ (i.e., $\kappa^{-1} \gg r_{c}$ ) dynamically interacting with the $l$-wave component of the three-dimensional continuum. The TDER theory extends effective range theory [22] for lowenergy elastic electron scattering from a short-range potential $U(r)$ to the case of a QQES. Within TDER theory, the wave function $\Phi_{\epsilon}(\mathbf{r}, t)$ outside the potential well $U(r)$ (i.e., for $r$ $\gtrsim r_{c}$ ) has an analytic form in terms of the retarded Green's function for a free electron in the field $\mathbf{F}(t), G\left(\mathbf{r}, t ; \mathbf{r}^{\prime}, t^{\prime}\right)$, and a periodic function, $f^{(l)}(t)$. The latter function enters the boundary condition for $\Phi_{\epsilon}(\mathbf{r}, t)$ inside the potential well $U(r)$ (i.e., for $r \lesssim r_{c}$ ) and satisfies the one-dimensional integrodifferential eigenvalue equation for the complex quasienergy $\epsilon$ (see Refs. [13,23] for details). Thus the TDER theory pro- 
vides an exactly solvable model for the multichannel ATD problem, treating electron-laser interactions exactly and electron-atom interactions as in effective range theory [22] (i.e., in terms of the scattering length $a_{l}$ and the effective range $r_{l}$ ).

Exact TDER results for the amplitude $\mathcal{A}_{n}\left(\mathbf{p}_{n}\right)$ and the corresponding differential ATD rates,

$$
\frac{d W_{n}\left(\mathbf{p}_{n}\right)}{d \Omega_{\mathbf{p}_{n}}} \equiv \Gamma_{n}\left(\mathbf{p}_{n}\right)=\frac{1}{m}\left|\sqrt{p_{n}} \mathcal{A}_{n}\left(\mathbf{p}_{n}\right)\right|^{2},
$$

have been derived in Refs. $[13,14]$ as expansions in generalized Bessel functions and the Fourier coefficients, $f_{2 k}^{(l)}$, of $f^{(l)}(t)$. However, in order to derive a closed-form analytic formula for the ATD amplitude $\mathcal{A}_{n}\left(\mathbf{p}_{n}\right)$, an alternative integral representation is more convenient and is the goal of this section. In carrying out these derivations, it is useful to note two important conclusions of our prior analysis [13,14]: (i) the partial ATD rate with zero projection $m$ of the angular momentum on the direction of linear laser polarization gives the dominant contribution to $\Gamma_{n}\left(\mathbf{p}_{n}\right)$; and (ii) for low frequencies $\left(\hbar \omega \ll\left|E_{0}\right|\right)$, the exact TDER results are in perfect agreement with those in the quasiclassical approximation.

For $s(l=0)$ and $p(l=1, m=0)$ states $\psi_{\kappa l m}(\mathbf{r})$, the TDER result for the QQES wave function $\Phi_{\epsilon}(\mathbf{r}, t)$ has the following form in the region $r \gtrsim r_{c}[13,23]$ :

$$
\Phi_{\epsilon}(\mathbf{r}, t)=\frac{C_{\kappa l}}{\kappa^{l}} \sqrt{\frac{2 l+1}{4 \pi}} \lim _{\mathbf{r}^{\prime} \rightarrow 0} \frac{\partial^{l}}{\partial z^{\prime l}} \chi\left(\mathbf{r}, \mathbf{r}^{\prime}, t\right),
$$

where

$$
\chi\left(\mathbf{r}, \mathbf{r}^{\prime} ; t\right)=-\frac{2 \pi \hbar^{2}}{m} \int_{-\infty}^{t} G\left(\mathbf{r}, t ; \mathbf{r}^{\prime}, t^{\prime}\right) f^{(l)}\left(t^{\prime}\right) e^{i \epsilon\left(t-t^{\prime}\right) / \hbar} d t^{\prime},
$$

and $C_{\kappa l}$ is the coefficient in the asymptotic form of $\psi_{\kappa l m}(\mathbf{r})$ at large distances,

$$
\left.\psi_{\kappa l m}(\mathbf{r})\right|_{r \gg \kappa^{-1}}=C_{\kappa l} \frac{e^{-\kappa r}}{r} Y_{l m}(\hat{\mathbf{r}}) .
$$

Using the spectral expansion of the Green's function in Eq. (3) (instead of the Feynman form in terms of the classical action, as used in Refs. [13,14,23]), the function $\chi\left(\mathbf{r}, \mathbf{r}^{\prime} ; t\right)$ has the form (cf. Appendix A)

$$
\chi\left(\mathbf{r}, \mathbf{r}^{\prime}, t\right)=\sum_{s} \frac{1}{T} \int_{0}^{T} \frac{e^{i \widetilde{\mathcal{S}}_{s}\left(\mathbf{r}, t, \mathbf{r}^{\prime}, t^{\prime}\right) / \hbar} f^{(l)}\left(t^{\prime}\right)}{\left|\mathbf{R}(\mathbf{r}, t)-\mathbf{R}\left(\mathbf{r}^{\prime}, t^{\prime}\right)\right|} d t^{\prime},
$$

where $T=2 \pi / \omega, p_{s}^{2} /(2 m)=\epsilon+s \hbar \omega-u_{p}$,

$$
\begin{aligned}
& \widetilde{\mathcal{S}}_{s}\left(\mathbf{r}, t ; \mathbf{r}^{\prime}, t^{\prime}\right)=-\int_{t^{\prime}}^{t}\left[\frac{m \dot{\mathcal{R}}^{2}(\tau)}{2}-u_{p}+s \hbar \omega\right] d \tau \\
&+p_{s}\left|\mathbf{R}(\mathbf{r}, t)-\mathbf{R}\left(\mathbf{r}^{\prime}, t^{\prime}\right)\right| \\
&+m\left[\mathbf{r} \cdot \mathcal{\mathcal { R }}(t)-\mathbf{r}^{\prime} \cdot \dot{\mathcal{R}}\left(t^{\prime}\right)\right], \\
& \mathcal{R}(t)=\mathbf{e}_{z} \frac{e F}{m \omega^{2}} \cos \omega t, \quad \mathbf{R}(\mathbf{r}, t)=\mathbf{r}-\mathcal{R}(t) .
\end{aligned}
$$

The asymptotic form of $\chi\left(\mathbf{r}, \mathbf{r}^{\prime}, t\right)$ at large $r$ is

$$
\begin{aligned}
\chi\left(\mathbf{r}, \mathbf{r}^{\prime}, t\right) \approx & e^{i \varphi(\mathbf{r}, t) / \hbar} \sum_{s} \frac{e^{i p_{s}|\mathbf{R}(\mathbf{r}, t)| \hbar-i s \omega t}}{r} \\
& \times \frac{1}{T} \int_{0}^{T} \exp \left\{\frac{i}{\hbar}\left[S\left(\mathbf{p}_{s}, t^{\prime}\right)-\mathbf{r}^{\prime} \cdot \mathbf{P}_{s}\left(t^{\prime}\right)\right]\right\} f^{(l)}\left(t^{\prime}\right) d t^{\prime},
\end{aligned}
$$

where $\mathbf{P}_{s}\left(t^{\prime}\right)=\mathbf{p}_{s}+m \dot{\mathcal{R}}\left(t^{\prime}\right)$,

$$
\begin{gathered}
\varphi(\mathbf{r}, t)=m \mathbf{r} \cdot \dot{\mathcal{R}}(t)-\int^{t}\left[\frac{m \dot{\mathcal{R}}^{2}(t)}{2}-u_{p}\right] d t, \\
S\left(\mathbf{p}_{s}, t\right)=\int^{t}\left[\frac{\left[\mathbf{p}_{s}+m \dot{\mathcal{R}}(t)\right]^{2}}{2 m}-\epsilon\right] d t .
\end{gathered}
$$

After substituting Eq. (7) into Eq. (2) and differentiating over $z^{\prime}$, the asymptotic behavior of $\Phi_{\epsilon}(\mathbf{r}, t)$ at $r \rightarrow \infty$ is

$$
\Phi_{\epsilon}(\mathbf{r}, t) \approx(-i)^{l} e^{i \varphi(\mathbf{r}, t) / \hbar} \sum_{s} \mathcal{A}_{s}^{(l)}\left(\mathbf{p}_{s}\right) \frac{e^{i p_{s}|\mathbf{R}(\mathbf{r}, t)| / \hbar-i s \omega t}}{r},
$$

where the $n$-photon ATD amplitude is expressed in the desired integral form:

$$
\begin{aligned}
\mathcal{A}_{n}^{(l)}\left(\mathbf{p}_{n}\right)= & C_{\kappa l} \sqrt{\frac{2 l+1}{4 \pi}} \frac{1}{T} \int_{0}^{T}\left[\frac{\mathbf{e}_{z} \cdot\left[\mathbf{p}_{n}+m \dot{\mathcal{R}}(t)\right]}{\hbar \kappa}\right]^{l} \\
& \times f^{(l)}(t) e^{i S\left(\mathbf{p}_{n}, t\right) / \hbar} d t .
\end{aligned}
$$

Note that by expanding $f^{(l)}(t)$ in Fourier series,

$$
f^{(l)}(t)=\sum_{k} f_{2 k}^{(l)} e^{-2 i k \omega t},
$$

the integral over $t$ in Eq. (9) may be evaluated analytically in terms of generalized Bessel functions and the result for $\mathcal{A}_{n}^{(l)}\left(\mathbf{p}_{n}\right)$ coincides with those in Refs. [13,14].

To simplify the notation, in what follows through the end of Sec. III we use scaled units (s.u.), in which energies, $\omega$, and momenta $(p)$ are measured in units of $\left|E_{0}\right|,\left|E_{0}\right| / \hbar$, and $\sqrt{2 m\left|E_{0}\right|}=\hbar \kappa$, respectively, and laser field amplitudes $F$ are given in units of $F_{0}=\sqrt{2 m\left|E_{0}\right|^{3}} / e \hbar=\left(F_{\mathrm{at}} / 2\right)\left(2\left|E_{0}\right| / E_{\mathrm{at}}\right)^{3 / 2}$, where $E_{\mathrm{at}}=m^{2} e^{4} / \hbar^{2} \approx 27.21 \mathrm{eV}$, and $F_{\mathrm{at}}=m^{2} e^{5} / \hbar^{4} \approx 5.14$ $\times 10^{9} \mathrm{~V} / \mathrm{cm}$. In these units, amplitude (9) becomes

$$
\begin{gathered}
\mathcal{A}_{n}^{(l)}\left(\mathbf{p}_{n}\right)=C_{\kappa l} \sqrt{\frac{2 l+1}{4 \pi}} \frac{1}{T} \int_{0}^{T}\left(\mathbf{e}_{z} \cdot \mathbf{p}_{n}-\frac{F}{\omega} \sin \omega t\right)^{l} \\
\times f^{(l)}(t) e^{i S\left(\mathbf{p}_{n}, t\right)} d t, \\
S\left(\mathbf{p}_{n}, t\right)=\int^{t}\left[\left(\mathbf{p}_{n}-\frac{\mathbf{F}}{\omega} \sin \omega t\right)^{2}-\epsilon\right] d t,
\end{gathered}
$$

where $\mathbf{F}=\mathbf{e}_{z} F$.

The simplest way to solve the integro-differential eigenvalue equation [for the function $f^{(l)}(t)$ and the complex quasienergy $\epsilon$ ] is to convert it to a system of linear homogeneous algebraic equations for the Fourier coefficients of $f^{(l)}(t)$. These equations have the following form $[13,14]$ : 


$$
\mathcal{R}_{l}(\epsilon+2 k \omega) f_{2 k}^{(l)}=\sum_{k^{\prime}} M_{k, k^{\prime}}^{(l)}(\epsilon) f_{2 k^{\prime}}^{(l)}
$$

where

$$
\mathcal{R}_{l}(E)=-a_{l}^{-1}+r_{l} E / 2-i E^{l+1 / 2},
$$

and $a_{l}$ and $r_{l}$ are the scattering length and effective range parameters. For bound-state problems, these parameters are intimately related to the binding energy $E_{0}$ and the coefficient $C_{\kappa l}$ in Eq. (4) [24]: e.g., for $s$ states, $1 / a_{0}=\kappa(1$ $\left.-\kappa r_{0} / 2\right)$ and $C_{0}^{2}=2 \kappa /\left(1-\kappa r_{0}\right)$. The explicit forms of the matrix elements $M_{k, k^{\prime}}^{(l)}(\epsilon)$ in terms of integrals involving Bessel functions are different for $l=0,1$. We present here the form for $s$ states $(l=0)$ (cf. Ref. [14] for $p$ states):

$$
\begin{aligned}
M_{k, k^{\prime}}^{(0)}(\epsilon)= & \frac{i^{k-k^{\prime}}}{\sqrt{4 \pi i}} \int_{0}^{\infty} \frac{d t}{t^{3 / 2}} e^{i\left[\epsilon+\left(k+k^{\prime}\right) \omega\right] t} \\
& \times\left\{e^{-i \lambda(t)} J_{k-k^{\prime}}[z(t)]-\delta_{k, k^{\prime}}\right\},
\end{aligned}
$$

where

$$
\begin{gathered}
z(t)=\frac{u_{p}}{\omega}\left(\sin \omega t-\frac{4 \sin ^{2}(\omega t / 2)}{\omega t}\right), \\
\lambda(t)=\frac{u_{p}}{\omega}\left(\omega t-\frac{4 \sin ^{2}(\omega t / 2)}{\omega t}\right) .
\end{gathered}
$$

Note also that $M_{k, k^{\prime}}^{(l)}(\epsilon)$ satisfies the following useful symmetry relation:

$$
M_{k+p, k^{\prime}+p}^{(l)}(\epsilon)=M_{k, k^{\prime}}^{(l)}(\epsilon+2 p \omega) .
$$

The function $f^{(l)}(t)$ is a key component of the TDER formulation of ATD since it comprises all information on the electron interaction with the core potential $U(r)$ during the ATD process. Thus, different approximations for the solution of system (13) for $f_{2 k}^{(l)}$ correspond to different approximate accounts of the potential $U(r)$ in ATD calculations. The three most important approximations for $f_{2 k}^{(l)}$ are [14]

$$
\begin{gathered}
f_{2 k}^{(l)}=\delta_{k, 0}, \\
f_{2 k}^{(l)}=M_{k, 0}^{(l)}\left(E_{0}\right), \quad k \neq 0, \\
f_{2 k}^{(l)}=\frac{M_{k, 0}^{(l)}\left(E_{0}\right)}{\mathcal{R}_{l}\left(E_{0}+2 k \omega\right)-M_{k, k}^{(l)}\left(E_{0}\right)} \\
\approx M_{k, 0}^{(l)}\left(E_{0}\right) \mathcal{R}_{l}^{-1}\left(E_{0}+2 k \omega\right), \quad k \neq 0 .
\end{gathered}
$$

All these approximations neglect the Stark effect (by approximating $\epsilon \approx E_{0}$ ), are valid in the low-frequency (or quasiclassical) limit $\left(\omega \ll\left|E_{0}\right|\right)$, and assume $f_{2 k}^{(l)}=1$ at $k=0$. They differ from one another in their account of binding potential effects for coefficients $f_{2 k}^{(l)}$ with $k \neq 0$. (For the case of the zero-range potential model, corresponding approximations are discussed in Refs. [11,25].)

Approximation (18) gives the strong-field approximation (or Keldysh) result for the amplitude $\mathcal{A}_{n}\left(\mathbf{p}_{n}\right), \mathcal{A}_{n}^{(K)}\left(\mathbf{p}_{n}\right)$, which follows from Eq. (11) upon making the substitutions $f^{(l)}(t) \rightarrow 1$ and $\epsilon \rightarrow E_{0}$. It describes only the low-energy part of the ATD spectrum. As is well known, this approximation accounts for the potential $U(r)$ only for the initial boundstate wave function. As discussed in Refs. [11,25], approximation (19) is equivalent to the "improved Keldysh approximation" (IKA) [5] within the TDER model, i.e., to a perturbative treatment of $U(r)$. As shown in Sec. III, IKA (19) is not appropriate for the correct evaluation of $\mathcal{A}_{n}\left(\mathbf{p}_{n}\right)$ in the tunneling limit. Instead, the more exact "rescattering approximation" (20) is necessary. As compared to Eq. (18), the rescattering approximation corresponds to the first-order perturbative account of the nondiagonal matrix elements $M_{k, k^{\prime}}^{(l)}$ in an iterative solution of system (13). The diagonal matrix elements $M_{k, k}^{(l)}\left(E_{0}\right)$ are neglected in the last equality in Eq. (20) since they vanish for $F \rightarrow 0$ and give negligible contribution to the denominator in Eq. (20) in the tunneling regime, $\gamma \ll 1$, where $\gamma$ is the Keldysh parameter, $\gamma=\omega / F$ $\left[=\left(\sqrt{2 m\left|E_{0}\right|} \omega\right) /(e F)\right.$ in absolute units $]$.

\section{ANALYTIC ANALYSIS OF THE ATD AMPLITUDE IN THE TUNNELING LIMIT}

\section{A. Analytic approximation for the function $f^{(l)}(t)$}

Rescattering approximation (20) for $f_{2 k}^{(l)}$ provides ATD rates that are in excellent agreement with exact TDER theory results over a wide interval of laser parameters (cf. Refs. $[13,14])$. To obtain an analytical approximation for the function $f^{(l)}(t)$ in Eq. (11), we thus start from the following approximate result (cf. Appendix B) for the matrix elements $M_{k, 0}^{(l)}\left(E_{0}\right) \equiv M_{k, 0}^{(l)}$ that enter expression (20) for $f_{2 k}^{(l)}$ :

$$
\begin{aligned}
M_{k, 0}^{(l)}= & \frac{2 l+1}{i^{l} \sqrt{16 \pi^{3} i}}\left(\frac{\omega}{\sqrt{F}}\right)^{3} \sum_{s} \int_{-\pi}^{\pi} \frac{e^{2 i k \xi}}{\sqrt{\cos \tau_{0}}} \\
& \times \frac{e^{i\left[\mathcal{S}\left(k_{s}, \tau_{0}\right)-\mathcal{S}\left(k_{s}, \xi\right)\right]}\left(k_{s}+\gamma^{-1} \sin \xi\right)^{l}}{\cos \xi-\cos \tau_{0}} d \xi,
\end{aligned}
$$

where $k_{s}=\sqrt{s \omega-1-u_{p}}$ and

$$
\mathcal{S}\left(k_{s}, \xi\right)=\frac{1}{\omega} \int^{\xi}\left[\left(k_{s}+\gamma^{-1} \sin \varphi\right)^{2}+1\right] d \varphi .
$$

The dimensionless time $\tau_{0}\left(=\omega t_{0}\right)$ in Eq. (21) is that root of the saddle-point equation,

$$
\frac{\partial \mathcal{S}\left(k_{s}, \tau_{0}\right)}{\partial \tau_{0}}=\left(k_{s}+\gamma^{-1} \sin \tau_{0}\right)^{2}+1=0,
$$

which has both a positive imaginary part and the smallest value of $\operatorname{Re} \tau_{0}$.

To obtain an analytical expression for $f^{(l)}(t)$, we use approximate result (20) for $f_{2 k}^{(l)}$ to evaluate the series in Eq. (10). For this purpose we obtain first the function $f^{(l)}(t)$ corresponding to IKA (19):

$$
f_{\mathrm{IKA}}^{(l)}(t)=1+\sum_{k \neq 0} M_{k, 0}^{(l)} e^{-2 i k \omega t} .
$$

Using Eq. (21) and the identity $\Sigma_{k} \exp [2 i k(\xi-\omega t)]=\pi \delta(\xi$ $-\omega t), f_{\text {IKA }}^{(l)}(\tau)$ may be presented as 


$$
\begin{aligned}
f_{\mathrm{IKA}}^{(l)}(t)-1= & \frac{2 l+1}{i^{l} \sqrt{16 \pi i}}\left(\frac{\omega}{\sqrt{F}}\right)^{3} \sum_{s} \frac{e^{i \mathcal{S}\left(k_{s}, \tau_{0}\right)}}{\sqrt{\cos \tau_{0}}} \\
& \times e^{-i \mathcal{S}\left(k_{s}, \tau\right)} \frac{\left(k_{s}+\gamma^{-1} \sin \tau\right)^{l}}{\cos \tau-\cos \tau_{0}},
\end{aligned}
$$

where $\tau=\omega t$. In the low-frequency limit, the sum over $s$ in Eq. (25) can be replaced by an integral over the active electron's momentum $q$ using the following substitutions:

$$
k_{s} \rightarrow q, \quad \sum_{s} \varphi\left(k_{s}\right) \rightarrow \frac{2}{\omega} \int_{0}^{\infty} \varphi(q) q d q .
$$

As a result, expression (25) has the form

$$
\begin{aligned}
f_{\mathrm{IKA}}^{(l)}(t)-1 \approx & \frac{(2 l+1) \omega^{2}}{i^{l} \sqrt{4 \pi i} F^{3 / 2}} \int \frac{q d q}{\sqrt{\cos \tau_{0}}} e^{i \mathcal{S}\left(q, \tau_{0}\right)} \\
& \times e^{-i \mathcal{S}(q, \tau)} \frac{\left(q+\gamma^{-1} \sin \tau\right)^{l}}{\cos \tau-\cos \tau_{0}} .
\end{aligned}
$$

Evaluating the integral in Eq. (27) by the saddle-point method, we obtain

$$
\begin{aligned}
f_{\text {IKA }}^{(l)}(t)-1 \approx & \frac{2 l+1}{2 i^{l+1}} \sqrt{\frac{\omega^{3}}{F \cos \tau_{0}\left(\tau-\tau_{0}\right)^{3}}} e^{i\left[\mathcal{S}\left(q, \tau_{0}\right)-\mathcal{S}(q, \tau)\right]} \\
& \times\left(q+\gamma^{-1} \sin \tau\right)^{l},
\end{aligned}
$$

where $\tau_{0}$ and $q$ satisfy the following system of equations:

$$
\begin{gathered}
q+\gamma^{-1} \sin \tau_{0}+i=0 \\
q=\frac{\cos \tau-\cos \tau_{0}}{\gamma\left(\tau-\tau_{0}\right)} .
\end{gathered}
$$

To obtain the function $f^{(l)}(t)$ corresponding to rescattering approximation $(20), f_{R}^{(l)}(t)$, we use a function, $g^{(l)}(t)$, as follows:

$$
f_{R}^{(l)}(t)-1=\left[f_{\text {IKA }}^{(l)}(t)-1\right] g^{(l)}(t) .
$$

The function $g^{(l)}(t)$ is smoothly varying compared to the oscillatory part of $f_{\text {IKA }}^{(l)}(t)$. It can be estimated using an identity following from Eqs. (20) and (31):

$$
M_{k, 0}^{(l)} \mathcal{R}_{l}^{-1}(2 k \omega-1)=\frac{1}{T} \int_{-T / 2}^{T / 2}\left[f_{\mathrm{IKA}}^{(l)}(t)-1\right] g^{(l)}(t) e^{2 i k \omega t} d t .
$$

As follows from Eq. (28), the saddle points for the integral in Eq. (32) are given by the equation

$$
\mathcal{E}\left(q, \tau_{j}\right)=2 k \omega-1,
$$

where $\mathcal{E}(q, \tau)$ is defined by

$$
\mathcal{E}(q, \tau)=\left(q+\gamma^{-1} \sin \tau\right)^{2} .
$$

Thus, Eq. (33) determines the moments of time $\tau_{j}$ at which the energy of the active electron, $\mathcal{E}\left(q, \tau_{j}\right)$, becomes equal to $2 k \hbar \omega+E_{0}$ (in absolute units). This is the energy resulting from an exchange of $2 k$ photons between the initially bound electron and the laser field, which results in the population of the $2 k$ th harmonic of the QQES wave function $\Phi_{\epsilon}(\mathbf{r}, t)$ for $r \lesssim r_{c}$. Equations (32) and (33) show that at the saddle points $t_{j}\left(=\tau_{j} / \omega\right)$ the function $g^{(l)}\left(t_{j}\right)$ coincides with $\mathcal{R}_{l}^{-1}\left(\mathcal{E}\left(q, \tau_{j}\right)\right)$. Thus we approximate $g^{(l)}(t)$ for any $t$ as follows:

$$
g^{(l)}(t)=\mathcal{R}_{l}^{-1}(\mathcal{E}(q, \tau))
$$

As a result, $f_{R}^{(l)}(t)$ in Eq. (31) has the form

$$
\begin{aligned}
f_{R}^{(l)}(t)= & 1+\frac{2 l+1}{2 i^{l+1}} \sqrt{\frac{\omega^{3}}{F \cos \tau_{0}\left(\tau-\tau_{0}\right)^{3}}} e^{i \mathcal{S}\left(q, \tau_{0}\right)} e^{-i \mathcal{S}(q, \tau)} \\
& \times[\mathcal{E}(q, \tau)]^{l / 2} \mathcal{R}_{l}^{-1}(\mathcal{E}(q, \tau))
\end{aligned}
$$

In agreement with the relation between the corresponding Fourier coefficients in Eqs. (20) and (19), $f_{R}^{(l)}(t)$ differs from $f_{\text {IKA }}^{(l)}(t)$ in Eq. $(28)$ only by the factor $\mathcal{R}_{l}^{-1}(\mathcal{E}(q, \tau))$. Equation (36) clearly shows that the integer unity on the right-hand side (rhs) corresponds to approximation (18) for $f_{2 k}^{(l)}$, while the second term describes rescattering effects. This "rescattering correction" to $f^{(l)}(t)$ is expressed in terms of two characteristic $\tau$-dependent parameters, $\tau_{0}$ and $q+\gamma^{-1} \sin \tau$, which have a simple quasiclassical interpretation. The time $\tau_{0}$ (in units of $\omega^{-1}$ ) is the time at which the initially bound electron appears in the continuum (due to tunneling) at some point $\mathbf{r}$ and starts to move along a closed trajectory under the action of the laser field $\mathbf{F}(t)$. The momentum $q+\gamma^{-1} \sin \tau$ is the electron's momentum upon returning to the same point $\mathbf{r}$ at time $\tau$ with kinetic energy $\mathcal{E}(q, \tau)$.

\section{B. Analytic result for the ATD amplitude}

Substituting $f_{R}^{(l)}(t)$ from Eq. (36) into integral (11), the ATD amplitude separates into a sum of "Keldysh" $\left[\mathcal{A}_{n}^{(K)}\left(\mathbf{p}_{n}\right)\right]$ and "rescattering" $\left[\widetilde{\mathcal{A}}_{n}^{(l)}\left(\mathbf{p}_{n}\right)\right]$ terms,

$$
\mathcal{A}_{n}^{(l)}\left(\mathbf{p}_{n}\right)=\mathcal{A}_{n}^{(K)}\left(\mathbf{p}_{n}\right)+\tilde{\mathcal{A}}_{n}^{(l)}\left(\mathbf{p}_{n}\right)
$$

The Keldysh amplitude is

$$
\mathcal{A}_{n}^{(K)}\left(\mathbf{p}_{n}\right)=\frac{C_{\kappa l}}{2 \pi} \sqrt{\frac{2 l+1}{4 \pi}} \int_{-\pi}^{\pi}\left(p_{\|}-\frac{F}{\omega} \sin \tau\right)^{l} e^{i S\left(\mathbf{p}_{n}, \tau\right)} d \tau,
$$

where $S\left(\mathbf{p}_{n}, \tau\right)$ is given by Eq. (12) after making the substitutions $\epsilon \rightarrow E_{0}=-1$ and $\omega t \rightarrow \tau$. The component of $\mathbf{p}_{n}$ parallel to the laser polarization axis is $p_{\|}=\mathbf{e}_{z} \cdot \mathbf{p}_{n}$, while $p_{\perp}$ $=\sqrt{\mathbf{p}_{n}^{2}-p_{\|}^{2}}$. Note that for the case of a linearly polarized field, the electron angular distributions are axially symmetric with respect to the laser polarization axis and are invariant under the reflection, $\theta \rightarrow(\pi-\theta)$. Thus, in what follows we assume that the electron ejection angle $\theta$ runs over the interval $(0, \pi / 2)$, i.e., $p_{\|} \geq 0$. The rescattering amplitude has then the following form: 


$$
\begin{aligned}
\tilde{\mathcal{A}}_{n}^{(l)}\left(\mathbf{p}_{n}\right)= & \frac{C_{\kappa l} l}{i^{l+1}}\left(\frac{2 l+1}{4 \pi}\right)^{3 / 2} \omega^{3 / 2} \int_{-\pi}^{\pi} \frac{e^{i \Phi(\tau)}}{\sqrt{F \cos \tau_{0}\left(\tau-\tau_{0}\right)^{3}}} \\
& \times\left(p_{\|}-\frac{F}{\omega} \sin \tau\right)^{l}[\mathcal{E}(q, \tau)]^{l / 2} \mathcal{R}_{l}^{-1}(\mathcal{E}(q, \tau)) d \tau,
\end{aligned}
$$

where

$$
\Phi(\tau)=S\left(\mathbf{p}_{n}, \tau\right)+\mathcal{S}\left(q, \tau_{0}\right)-\mathcal{S}(q, \tau) .
$$

The saddle points of the integrand in Eq. (39) are given by the equation $\partial \Phi(\tau) / \partial \tau=0$, i.e.,

$$
\Phi^{\prime}(\tau)=\frac{1}{\omega}\left[\left(\mathbf{p}_{n}-\mathbf{e}_{z} \frac{F}{\omega} \sin \tau\right)^{2}-\mathcal{E}(q, \tau)\right]=0,
$$

where the derivative $\Phi^{\prime}(\tau)$ may be parametrized as follows:

$$
\begin{aligned}
\Phi^{\prime}(\tau) & =\frac{1}{\omega}\left[\mathbf{p}_{n}-\mathbf{e}_{z} q_{+}(\tau)\right] \cdot\left(\mathbf{p}_{n}+\mathbf{e}_{z} q\right) \\
& =\frac{1}{\omega}\left[\mathbf{p}_{n}^{2}-2 \gamma^{-1} p_{\|} \sin \tau-q_{+}(\tau) q\right],
\end{aligned}
$$

where

$$
q_{+}(\tau)=q+2 \gamma^{-1} \sin \tau .
$$

Equations (29), (30), and (41) give a coupled system of saddle-point equations for $\tau_{0}, q$, and $\tau$. However, the proper saddle-point evaluation of the integral in Eq. (39) leads to a complicated expression for the ATD amplitude as a sum of separate saddle-point contributions (cf. [2]). Moreover, the standard saddle-point method becomes inapplicable at the classical cutoff energy $\left(\mathbf{p}_{n}^{2}=10 u_{p}\right)$, where two isolated saddle points of the function $\Phi(\tau)$ merge into a single one [9].

In this paper we perform an approximate evaluation of the integral over $\tau$ in Eq. (39) based on the general ideas of the uniform approximation [26]. Namely, we approximate the function $\Phi(\tau)$ by a cubic polynomial near the point $\tau=\bar{\tau}$, where $\Phi^{\prime \prime}(\bar{\tau})=0$ and, as discussed below, $\Phi^{\prime}(\tau)$ has a maximum. Moreover, we consider only classically allowed closed electron trajectories, in which case $\tau_{0}, q$, and $\bar{\tau}$ are real. As will be shown, our approximation is appropriate for the description of the high-energy part of the ATD spectrum. (A similar method for estimating $\mathcal{A}_{n}^{(l=0)}\left(\mathbf{p}_{n}\right)$ was used in Ref. [9], in which the authors started with a modified Keldysh result for the ATI amplitude [taking into account the potential $U(r)$ perturbatively]. A more accurate semianalytical description of the ATI plateau using the uniform approximation within the framework of the IKA was presented in Ref. [8].)

We consider first Eqs. (29), (30), and (41) in the lowest order in $\gamma$. In this case, $\tau_{0} \approx \widetilde{\tau}_{0}$, while $q \approx \widetilde{q}=-\gamma^{-1} \sin \widetilde{\tau}_{0}$, as follows from Eq. (29). Substituting $q \rightarrow \tilde{q}$ in Eq. (30), we obtain a relation between the dimensionless times $\widetilde{\tau}_{0}$ and $\tau$ in the lowest (i.e., zero) order in $\gamma$ :

$$
\sin \widetilde{\tau}_{0}=-\frac{\cos \tau-\cos \widetilde{\tau}_{0}}{\tau-\widetilde{\tau}_{0}} .
$$

As an analysis of the explicit form for $\Phi^{\prime \prime}(\tau)$ shows, the first derivative, $\Phi^{\prime}(\tau)$, achieves its maximum near the point $\tau$ $=\tilde{\tau}$, where $q_{+}(\tilde{\tau})$ is a maximum. The positions of the maxima of $q_{+}(\tau)$ are given by the equation $q_{+}^{\prime}(\tau)=0$, which [using Eqs. (30), (43), and (44)] may be represented as

$$
2 \cos \tau-\frac{\sin \tau-\sin \tilde{\tau}_{0}}{\tau-\widetilde{\tau}_{0}}=0 .
$$

Numerical analysis of Eqs. (44) and (45) shows that $q_{+}(\tau)$ attains its global maximum at $\tilde{\tau}_{0}=-2.881, \tau=\tilde{\tau}=1.425$ : $\max \left\{q_{+}(\tau)\right\}=q_{+}(\widetilde{\tau})=2.24 \gamma^{-1}$. The physical meaning of the times $\widetilde{\tau}_{0}$ and $\widetilde{\tau}$ becomes clear if one represents saddle-point equation (41) as [cf. Eq. (42)]

$$
\left[\mathbf{p}_{n}-\mathbf{e}_{z} q_{+}(\tau)\right] \cdot\left(\mathbf{p}_{n}+\mathbf{e}_{z} q\right)=0,
$$

and considers the case of $\mathbf{p}_{n} \| \mathbf{e}_{z}$. For this case Eq. (46) has the solution $\left|\mathbf{p}_{n}\right|=q_{+}(\widetilde{\tau})$, where $q_{+}^{2}(\widetilde{\tau}) \approx 10 u_{p}$. The times $\widetilde{\tau}_{0}$ and $\widetilde{\tau}$, therefore, are the saddle points of Eqs. (29), (30), and (41) for the case when the electron is ejected along the polarization axis $(\theta=0)$ with the maximum classically allowed energy, $10 u_{p}$ (i.e., the cutoff energy).

Near the point $\tau=\widetilde{\tau}$, the function $\Phi^{\prime}(\tau)$ in Eq. (42) can be approximated by a three-term quadratic polynomial in $x$ $=(\tau-\tilde{\tau}) / \gamma, a x^{2}+b x+c$, by expanding $q_{+}(\tau)$ and $q$ in $\tau-\tilde{\tau}$ up to quadratic terms. The linear term in $x$ may be removed by making the linear transformation $\tilde{\tau} \rightarrow[\bar{\tau}-b /(2 a)]$. As a result, the expansion of $\Phi^{\prime}(\tau)$ near its maximum (at $\tau=\bar{\tau}$ ) has the following form:

$$
\Phi^{\prime}(\tau)=\frac{1}{\omega}\left[\frac{a(\tau-\bar{\tau})^{2}}{\gamma^{2}}-\frac{b^{2}}{4 a}+c\right],
$$

where

$$
\begin{gathered}
\bar{\tau}=\tilde{\tau}+b /(2 a), \\
a=\gamma p_{\|}+\alpha_{0}, \\
b=\alpha_{1}\left(p_{\|}-\tilde{q}_{+}\right), \\
c=\left(\mathbf{p}_{n}-\Delta \mathbf{p}\right)^{2}-\mathcal{E}(\tilde{q}, \tilde{\tau}) \approx\left(\mathbf{p}_{n}-\Delta \mathbf{p}\right)^{2}-3.11 u_{p}, \\
\Delta \mathbf{p}=\mathbf{F} \frac{\sin \tilde{\tau}}{\omega}=0.989 \frac{\mathbf{F}}{\omega} \approx \frac{\mathbf{F}}{\omega} .
\end{gathered}
$$

The numerical values of $q_{+}(\tau)$ and $q$ at $\tau=\tilde{\tau}$ and of the parameters $\quad \alpha_{0}$ and $\alpha_{1}$ are $q_{+}(\widetilde{\tau}) \equiv \tilde{q}_{+}=2.24 \gamma^{-1}, \quad q(\widetilde{\tau}) \equiv \tilde{q}$ $=0.26 \gamma^{-1}, \alpha_{0}=0.10$, and $\alpha_{1}=0.29$. According to Eq. (47), the function $\Phi(\tau)$ near the point $\tau=\bar{\tau}$ is approximated by a cubic polynomial:

$$
\Phi(\tau)=\frac{1}{\omega}\left[\frac{a(\tau-\bar{\tau})^{3}}{3 \gamma^{2}}+\left(c-\frac{b^{2}}{4 a}\right)(\tau-\bar{\tau})\right]+\Phi_{0},
$$

where $\Phi_{0}$ is given by [cf. Eq. (40)]

$$
\Phi_{0}=S\left(\mathbf{p}_{n}, \bar{\tau}\right)+\mathcal{S}\left(\widetilde{q}, \widetilde{\tau}_{0}\right)-\mathcal{S}(\tilde{q}, \bar{\tau}) .
$$

As our numerical analysis shows, lowest-order (in $\gamma$ ) result (48) for $\Phi(\tau)$ is not sufficiently accurate for the evaluation of the integral in Eq. (39). To consider the next-order corrections in $\gamma$ for the coefficients $a, b$, and $c$, it is necessary 
first to analyze more accurately the system of saddle-point equations (29) and (30), beyond the approximations given by $q=-\gamma^{-1} \sin \widetilde{\tau}_{0}$ and Eq. (44), both of which were used for the derivation of Eq. (47). By an iterative (in $\gamma$ ) solution of Eqs. (29) and (30), starting from $\tau_{0}=\widetilde{\tau}_{0}(\tau)$ and $q=-\gamma^{-1} \sin \widetilde{\tau}_{0}(\tau)$, we find the corrections of order $\gamma$ to $\tau_{0}$ and $q$ :

$$
\begin{gathered}
\tau_{0}=\widetilde{\tau}_{0}-i \gamma / \cos \widetilde{\tau}_{0}, \\
q \approx-\gamma^{-1} \sin \widetilde{\tau}_{0}-\frac{\gamma}{2\left(\tau-\tilde{\tau}_{0}\right) \cos \widetilde{\tau}_{0}} .
\end{gathered}
$$

Equations (50) and (51) allow one to obtain $\gamma$-dependent corrections to factors $a, b, c$ in Eq. (47). However, numerical analysis shows that the $\gamma^{2}$ corrections to $a$ and $b$ give negligible contributions to the final results, so that only the correction to the parameter $c, c \rightarrow c+\Delta$, where

$$
\Delta=2 \cos \tilde{\tau} / \cos \tilde{\tau}_{0} \approx-0.30,
$$

is significant. [In fact, $-\Delta=0.30$ gives the first quantum correction to the classical result for the energy $\mathcal{E}$, where $\mathcal{E}_{\mathrm{cl}}$ $\left.\equiv \mathcal{E}(\tilde{q}, \widetilde{\tau}) \approx 3.11 u_{p}.\right]$ Also, the imaginary $\gamma$-dependent term on the rhs of Eq. (50) contributes to $\Phi_{0}$ in Eq. (49). As a result, $\Phi_{0}$ becomes complex. Replacing $\widetilde{\tau}_{0}$ in Eq. (49) by the more accurate result (50), we obtain the lowest (first order in $\gamma)$ correction to $\Phi_{0}$ :

$$
\Delta \Phi_{0}=\frac{2 i}{3 F\left|\cos \tilde{\tau}_{0}\right|} .
$$

Approximating $\Phi(\tau)$ in Eq. (39) by Eq. (48) (with $c \rightarrow c$ $\left.+\Delta, \Phi_{0} \rightarrow \Phi_{0}+\Delta \Phi_{0}\right)$, the integral over $\tau$ may be estimated analytically. Note first that the behavior of the integrand near the saddle points governs the value of integrals involving highly oscillatory exponential functions, such as in Eq. (39). In the lowest order in $\gamma$, the saddle points of the function $\Phi(\tau), \tau_{ \pm}\left(\mathbf{p}_{n}\right)$, are given by substituting Eq. (47) for $\Phi^{\prime}(\tau)$ into saddle-point equation (41):

$$
\tau_{ \pm}\left(\mathbf{p}_{n}\right)=\bar{\tau} \pm \frac{\gamma}{2 a} \sqrt{b^{2}-4 a c} .
$$

One sees that the saddle points $\tau_{+}$and $\tau_{-}$are symmetric with respect to $\bar{\tau}$ for any $\mathbf{p}_{n}$. According to Eq. (41), the following substitution for the energy $\mathcal{E}(q, \tau)$ in the integrand of Eq. (39) is valid at the saddle points:

$$
\mathcal{E}(q, \tau) \rightarrow\left(\mathbf{p}_{n}-\mathbf{e}_{z} \frac{F}{\omega} \sin \tau_{ \pm}\left(\mathbf{p}_{n}\right)\right)^{2} .
$$

As follows from the discussion below Eq. (46) and as a direct analysis of Eq. (54) shows, the saddle points $\tau_{ \pm}$merge to a single one, $\tau_{ \pm} \rightarrow \tilde{\tau}$, at the classical cutoff energy, i.e., at $\mathbf{p}_{n}^{2}=5 \gamma^{-2}=10 u_{p}$ and $\theta=0$. Thus in order to have a simple analytic expression for the ATD amplitude, we approximate the saddle points $\tau_{ \pm}$in Eq. (55) by $\widetilde{\tau}$ and replace $\tau_{0}$ and $\tau$ in the smooth part of the integrand in Eq. (39) by $\widetilde{\tau}_{0}$ and $\tilde{\tau}$. (As our numerical results show, this approximation is reasonable for approximating the ATD rates for photoelectron energies $E_{n} \equiv \mathbf{p}_{n}^{2} \gtrsim 7 u_{p}$.) Finally, we extend the integration limits in the integral in Eq. (39) to $\pm \infty$, so that the integral of $\exp [i \Phi(\tau)]$ may be expressed in terms of the Airy function $\operatorname{Ai}(\xi)$. As a result, the ATD amplitude $\widetilde{\mathcal{A}}_{n}^{(l)}\left(\mathbf{p}_{n}\right)$ takes the following analytic form:

$$
\begin{aligned}
\tilde{\mathcal{A}}_{n}^{(l)}\left(\mathbf{p}_{n}\right)= & \frac{C_{\kappa l}}{i^{l+1}} \sqrt{\frac{(2 l+1) \omega^{5}}{16 \pi a^{2 / 3} F^{7 / 3} \cos \widetilde{\tau}_{0}\left(\tilde{\tau}-\widetilde{\tau}_{0}\right)^{3}}} e^{i \Phi_{0}} \\
& \times e^{-2 /\left(3 F\left|\cos \widetilde{\tau}_{0}\right|\right)} \operatorname{Ai}(\widetilde{\xi}) f^{(l)}\left(\mathbf{p}_{n}-\Delta \mathbf{p}\right),
\end{aligned}
$$

where

$$
\begin{gathered}
\Delta \mathbf{p}=\mathbf{e}_{z} \frac{F}{\omega} \sin \tilde{\tau} \approx \mathbf{e}_{z} \frac{F}{\omega}, \\
\widetilde{\xi}=\left(a F^{2}\right)^{-1 / 3}\left[c+\Delta-b^{2} /(4 a)\right], \\
f^{(l)}(\mathbf{p})=(2 l+1) p^{l}\left(\mathbf{p} \cdot \mathbf{e}_{z}\right)^{l} \mathcal{R}_{l}^{-1}\left(p^{2}\right) .
\end{gathered}
$$

\section{RESULTS AND DISCUSSION}

\section{A. Three-step formula for the ATD rates $\Gamma_{n}^{(R)}\left(\mathbf{p}_{n}\right)$}

Converting $\widetilde{\mathcal{A}}_{n}^{l}\left(\mathbf{p}_{n}\right)$ in Eq. (56) from scaled to absolute units and substituting the result into Eq. (1), the $n$-photon differential ATD rate $\Gamma_{n}^{(R)}\left(\mathbf{p}_{n}\right)$ in the high-energy plateau region takes the following analytic form:

$$
\Gamma_{n}^{(R)}\left(\mathbf{p}_{n}\right)=\mathcal{I}(F, \omega) \mathcal{W}\left(\mathbf{p}_{n}\right) \sigma^{(l)}\left(\mathbf{p}_{n}-\Delta \mathbf{p}\right) .
$$

Thus, our key results, Eqs. (56) and (59), factorize into a product of three terms corresponding to the three-step rescattering model of ATI or ATD, thus providing a convincing quantum justification for this model. Below we discuss each of the three terms in Eq. (59) separately. by

The "ionization factor" $\mathcal{I}(F, \omega)$ (in units of $\left.\mathrm{cm}^{-1}\right)$ is given

$$
\mathcal{I}(F, \omega)=C_{\kappa l}^{2} \frac{2 l+1}{8 \pi}\left(\frac{\hbar \omega}{\left|E_{0}\right|}\right)^{2} \frac{F_{0}}{F} e^{-4 F_{0} /(3 F)},
$$

where, more exactly, the field amplitude $F$ should be replaced by $\tilde{F}=F\left|\cos \widetilde{\tau}_{0}\right|$ [cf. Eq. (56)], i.e., by the magnitude of the laser field $\mathbf{F}(t)$ at the moment of ionization, $t=\widetilde{\tau}_{0} / \omega$. (This is a consequence of our approximation $\gamma \ll 1$, which is equivalent to the quasistatic limit.) However, for simplicity, we approximate $\widetilde{F} \approx F$, because $\left|\cos \widetilde{\tau}_{0}\right|=0.966$. As shown in Appendix $\mathrm{C}$, the factor $\mathcal{I}(F, \omega)$ is given by the modulus squared of Keldysh amplitude (38) in the quasiclassical limit $(\gamma \rightarrow 0)$ for the case of low-energy $\left(\left|\mathbf{p}_{n}\right| \rightarrow 0\right)$ electrons ejected along the direction of polarization of the laser field $\mathbf{F}(t)$. The factor $\mathcal{I}(F, \omega)$ may also be represented (cf. Appen$\operatorname{dix} C)$ in terms of the decay rate $\Gamma_{\mathrm{st}}(F)$ of an initial bound state $\psi_{\kappa l m}(\mathbf{r})$, with $m=0$ in a static electric field $\mathbf{F}$ :

$$
\mathcal{I}(F, \omega)=\left.2 \pi\left|\mathcal{A}_{n}^{(K)}\left(\mathbf{p}_{n}\right)\right|^{2}\right|_{p_{n}=0}=\frac{m}{\pi \hbar \kappa} \gamma^{2} \Gamma_{\mathrm{st}}(F),
$$

where $\Gamma_{\mathrm{st}}(F)$ is given by [27]

$$
\Gamma_{\mathrm{st}}(F)=\frac{\hbar \kappa}{8 m}(2 l+1) C_{\kappa l}^{2} \frac{F}{F_{0}} e^{-4 F_{0} /(3 F)} .
$$


The "propagation factor" $\mathcal{W}\left(\mathbf{p}_{n}\right)$ (in units of $\mathrm{cm}^{-1} \mathrm{~s}^{-1}$ ) describes the propagation of an electron in the laser-dressed continuum from the moment of ionization $t=t_{i}=\widetilde{\tau}_{0} \omega^{-1}$ up to the rescattering event, at $t=t_{r}=\widetilde{\tau} \omega^{-1}$ :

$$
\mathcal{W}\left(\mathbf{p}_{n}\right)=\frac{p_{n} \omega}{\hbar\left(\widetilde{\tau}-\widetilde{\tau}_{0}\right)^{3}}\left(\frac{\hbar \omega}{E_{\mathrm{at}}}\right)^{2} \frac{\mathrm{Ai}^{2}(\xi)}{\left[a I /\left(2 I_{\mathrm{at}}\right)^{2 / 3}\right.},
$$

where $\left(\widetilde{\tau}-\widetilde{\tau}_{0}\right)^{3}=79.84 \approx 80$,

$$
\begin{gathered}
\xi=\frac{\left|\mathbf{p}_{n}-\Delta \mathbf{p}\right|^{2} /(2 m)-\left(3.11 u_{p}+0.3\left|E_{0}\right|\right)}{E_{\mathrm{at}}\left[a I /\left(2 I_{\mathrm{at}}\right)\right]^{1 / 3}}, \\
a=\frac{p_{\|} \omega}{e F}+\alpha_{0}, \quad \Delta \mathbf{p}=\frac{e}{\omega} \mathbf{F},
\end{gathered}
$$

and $I_{\mathrm{at}}=3.51 \times 10^{16} \mathrm{~W} / \mathrm{cm}^{2}$. We have neglected the term $\sim b^{2}$ / (4a) [cf. Eq. (57)] in Eq. (64) for $\xi$ because its contribution is considerable only for $\theta \gtrsim \pi / 4$, where $\Gamma_{n}^{(R)}\left(\mathbf{p}_{n}\right)$ is a few orders of magnitude smaller than for $\theta=0$. Within this approximation, the numerator in the Airy function argument $\xi$ [cf. Eq. (64)] has a transparent physical meaning: it is the difference between the kinetic energy of an electron in a laser field at the moment of rescattering, $t=t_{r}$, and that acquired during its propagation in the laser field over the interval $\delta \tau=\widetilde{\tau}-\widetilde{\tau}_{0}=4.306$. Note that the latter kinetic energy involves the quantum correction $0.3\left|E_{0}\right|$, while its "classical" part, $\mathcal{E}(\widetilde{q}, \widetilde{\tau}) \approx 3.11 u_{p}$, is slightly smaller than the corresponding energy $3.17 u_{p}$ for the case of harmonic generation.

The last factor in Eq. (59) is the cross section for elastic scattering of the electron, $\left|\mathbf{p}_{i}\right|=\left|\mathbf{p}_{f}\right|$, where the initial momentum $\mathbf{p}_{i}$ is directed along the laser polarization, $\mathbf{p}_{i} \equiv \mid \mathbf{p}_{n}$ $-\Delta \mathbf{p} \mid \mathbf{e}_{z}$, and the final momentum is given by $\mathbf{p}_{f} \equiv \mathbf{p}_{n}-\Delta \mathbf{p}$. In terms of the wave vector $\mathbf{k}=\mathbf{p} / \hbar, \sigma^{(l)}(\mathbf{p})$ has the form

$$
\sigma^{(l)}(\mathbf{p})=\frac{(2 l+1)^{2} k^{2 l}\left(\mathbf{k} \cdot \mathbf{e}_{z}\right)^{2 l}}{\left|\mathcal{R}_{l}\left(k^{2}\right)\right|^{2}}=\frac{(2 l+1)^{2} k^{2 l}\left(\mathbf{k} \cdot \mathbf{e}_{z}\right)^{2 l}}{\left(-\frac{1}{a_{l}}+\frac{r_{l} k^{2}}{2}\right)^{2}+k^{2(2 l+1)}} .
$$

We emphasize that Eq. (66) gives the exact elastic cross section within effective range theory for $l$-wave scattering, in which case the scattering phase $\delta_{l}(k)$ in the $l$-wave part of the elastic-scattering amplitude [22],

$$
f_{l}(\mathbf{p})=\frac{2 l+1}{2 i k}\left(e^{2 i \delta_{l}}-1\right) P_{l}(\cos \theta), \quad \cos \theta=\left(\hat{\mathbf{k}} \cdot \mathbf{e}_{z}\right),
$$

where $P_{l}(x)$ is a Legendre polynomial, is parametrized according to effective range theory [22] as

$$
k^{2 l+1} \cot \delta_{l}(k)=-\frac{1}{a_{l}}+\frac{r_{l} k^{2}}{2} .
$$

It is worthwhile noting that the factor $\mathcal{R}_{l}(E)$ in TDER theory [cf. Eq. (13)] is intimately related to the dynamical part of the amplitude $f_{l}(\mathbf{p})$ for electron scattering from a short-range potential.

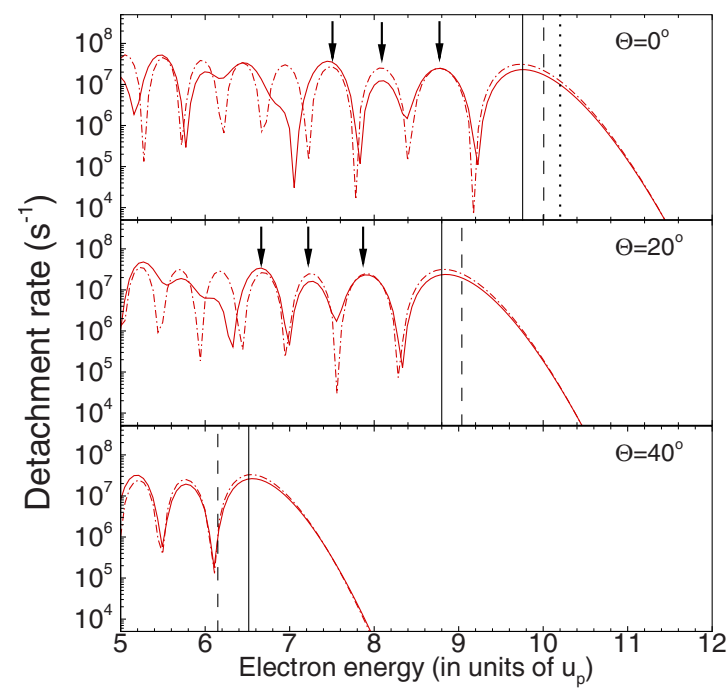

FIG. 1. (Color online) High-energy ATD spectra for three different angles $\theta$ for $\mathrm{H}^{-}$in a $\mathrm{CO}_{2}$ laser field $(\lambda=10.6 \mu \mathrm{m})$ with intensity $I=2 \times 10^{12} \mathrm{~W} / \mathrm{cm}^{2}(\gamma=0.424)$. Solid curves: exact TDER results; dot-dashed curves: analytic result (59). Vertical lines mark the positions of the plateau cutoff according to Eq. (73) (solid lines), Eq. (72) (dashed lines), and Eq. (71) (dotted line). The arrows mark the positions of the maxima of the interference oscillations according to Eq. (83).

Equation (59) for $\Gamma_{n}^{(R)}\left(\mathbf{p}_{n}\right)$ shows that the electron wave packet $W(E)$ introduced in Refs. [15-17] has the following explicit analytic structure:

$$
W(E)=\mathcal{I}(F, \omega) \mathcal{W}\left(\mathbf{p}_{n}\right) .
$$

Moreover, the elastic cross section $\sigma^{(l)}$ in Eq. (59) depends on the same "shifted" momentum, $\mathbf{p}_{n}-\Delta \mathbf{p}$, that was introduced in the empirical parametrization for ATI or ATD rates in Refs. [15-17]. Note also that a closed-form analytic formula (involving an Airy function) for ATI or ATD angular distributions near the plateau cutoff was obtained earlier in Ref. [9] for a ground state of $s$ symmetry without specification of any particular atomic potential $U(r)$, which was taken into account perturbatively, in first order. The differences from our expression (59) are: (i) $\Gamma_{n}^{(R)}\left(\mathbf{p}_{n}\right)$ in Ref. [9] involves the Born result $\left[\sim\left|U\left(\mathbf{p}_{n}\right)\right|^{2}\right]$ for the elastic cross section with an "unshifted" (i.e., $\Delta \mathbf{p}=\mathbf{0}$ ) momentum $\mathbf{p}_{n}$, and (ii) the argument of the Airy function in Ref. [9] is different from that in our Eqs. (57) and (64): actually the authors of Ref. [9] did not present an explicit analytic expression for this argument, but (based on results of numerical analyses) approximated this argument by a term proportional to $\left[\mathbf{p}_{n}^{2} /(2 m)-E_{c}^{\mathrm{cl}}(\theta)\right]$, where $E_{c}^{\mathrm{cl}}(\theta)$ is the classical position of the plateau cutoff for a given ejection angle $\theta$ [cf. Eq. (72)].

Figures 1 and 2 compare the results of our analytic formula (56) for the ATD amplitude with results of an exact numerical evaluation of amplitude (9) expressed in terms of generalized Bessel functions $[13,14]$. For both the $\mathrm{H}^{-}$and $\mathrm{F}^{-}$ negative ions, one sees that for detached electron energies at the high-energy end of the ATD plateau, as well as beyond the plateau cutoff, the analytic and exact numerical results agree quantitatively very well. Our analytical results not only 


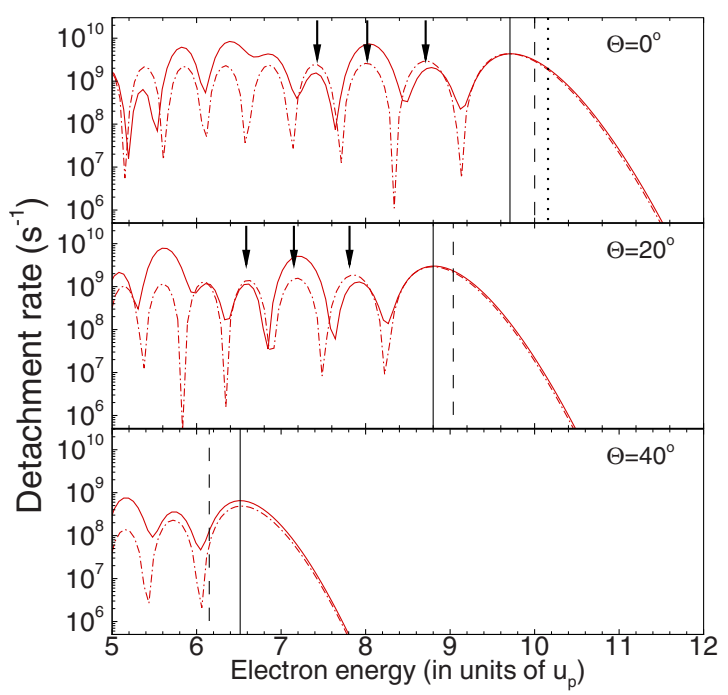

FIG. 2. (Color online) The same as in Fig. 1, but for $\mathrm{F}^{-}$in a laser field with $\lambda=1.8 \mu \mathrm{m}$ and $I=4 \times 10^{13} \mathrm{~W} / \mathrm{cm}^{2}(\gamma=0.375)$.

correctly predict the differential ATD rates $\Gamma_{n}^{(R)}\left(\mathbf{p}_{n}\right)$ for electrons ejected along the laser polarization axis $(\theta=0)$, but also reproduce well the shape of the angular distributions in the $\theta$-dependent cutoff regions of the ATD spectra. It is worthwhile emphasizing that the shift $\Delta \mathbf{p}_{n}$ of the momentum $\mathbf{p}_{n}$ in the argument of the elastic-scattering cross section in Eq. (59) affects significantly the magnitude of the ATD rates: neglecting this shift reduces the absolute value of the rates by about an order of magnitude compared to the exact TDER results in Figs. 1 and 2. This reduction is not surprising since $\sigma^{(l)}(\mathbf{p})$ increases with decreasing electron energy. Note that the evolution of the angular distribution $\Gamma_{n}\left(\mathbf{p}_{n}\right)$ as $n$ varies across the cutoff region [i.e., the transition from an angular distribution $\Gamma_{n}\left(\mathbf{p}_{n}\right)$ concentrated along the vector $\mathbf{F}(t)$ at the cutoff energy and beyond to the side-lobe structure existing for below-cutoff energies] was described in detail in Ref. [9]. Our results are in good qualitative agreement with those in Ref. [9]. Hence we do not pursue this question in the present paper.

In the following subsections we discuss in turn a number of very general results for the ATI or ATD process that are obtained using analytic formulas (56) and (59).

\section{B. ATI or ATD cutoff position}

The well-known classical position of the ATI or ATD cutoff (for $\theta=0$ ),

$$
E_{c}^{\mathrm{cl}}=10.007 u_{p} \approx 10 u_{p}
$$

has been obtained from numerical solution of the classical Newton equations for a free electron in a laser field within the rescattering scenario [28]. The first quantum correction to classical law (70),

$$
E_{c}^{(q)}=10 u_{p}+0.538\left|E_{0}\right|,
$$

was found in Ref. [29] using the strong-field approximation and saddle-point analyses in terms of quasiclassical electron trajectories. As in the case of the cutoff energy in high-order harmonic generation (where the quantum correction is about $\left.0.32\left|E_{0}\right|[30]\right)$, the quantum corrections increase the extent of the plateaus. However, as shown by numerical results, the position of the last maximum in both ATI and harmonic generation spectra, after which rates start to decrease steeply, may take place both above and below the classically predicted cutoff positions. Moreover, the above-mentioned (quasi)classical derivations define the cutoff position indirectly, not as the maximum in the rate before it begins decreasing, but as the maximum energy of an electron moving in a laser field along a one-dimensional trajectory. Therefore, the three-dimensional structure of the electron wave packet as well as its transverse spreading is ignored in such considerations. In contrast, our analytical results for ATD rates show excellent agreement of the cutoff positions with those of exact numerical results (cf. Figs. 1 and 2), thus offering the possibility of predicting the cutoff position analytically, as the energy at which $\Gamma_{n}^{(R)}\left(\mathbf{p}_{n}\right)$ attains its highest energy maximum. In addition, our analysis allows us to obtain the dependence of the cutoff energy on the ejection angle $\theta$. To the best of our knowledge, an analytical approximation for this dependence at small $\theta$ was suggested for the first time in Ref. [9]:

$$
E_{c}^{\mathrm{cl}}(\theta)=E_{c}^{\mathrm{cl}}-7.9 u_{p} \theta^{2}=\left(10-7.9 \theta^{2}\right) u_{p},
$$

where the ejection angle $\theta$ is in radians. Note that our analytic formulas, derived below, for the plateau cutoff energies for $\theta=0$ [cf. Eq. (79)] and $\theta>0$ [cf. Eq. (80)] differ from those in Eq. (71) [29] and Eq. (72) [9], respectively, that have been obtained previously by others.

According to the quasiclassical considerations of Ref. [28], the extent of the high-energy plateau is determined by the dynamics of the rescattering wave packet, which in our case is given by the factor $\mathcal{W}\left(\mathbf{p}_{n}\right)$ in Eq. (63). Therefore, we define the ATI or ATD cutoff as the highest energy maximum of the function $\mathcal{W}\left(\mathbf{p}_{n}\right)$ [corresponding to $\left|\mathbf{p}_{n}\right|=p_{c}(\theta)$ ] for a given direction of the vector $\mathbf{p}_{n}$, i.e., a given $\theta$. Since the Airy function $\operatorname{Ai}(\xi)$ decreases rapidly for positive values of the argument $\xi$ and oscillates for $\xi<0$, the equation for the plateau cutoff momentum $p_{c}(\theta)$ and the corresponding cutoff energy, $E_{c}(\theta)=p_{c}^{2}(\theta) /(2 m)$, follows by equating the argument of $\operatorname{Ai}(\widetilde{\xi})$ in Eq. (56) to the position $\xi_{1}$ of the first maximum of $\operatorname{Ai}(\xi)$ for $\xi<0$ (at $\xi_{1} \approx-1.019$; see, e.g., Ref. [31]):

$$
\widetilde{\xi} \equiv\left(\frac{2 I_{\mathrm{at}}}{a I}\right)^{1 / 3} \frac{1}{E_{\mathrm{at}}}\left[c+\Delta\left|E_{0}\right|-\frac{b^{2}}{4 a}\right]=\xi_{1},
$$

where the dimensionless parameter $a$ is defined by Eq. (65) and the parameters $c$ and $b^{2}$ in absolute units are given by

$$
\begin{gathered}
c=\frac{\left(\mathbf{p}_{n}-\Delta \mathbf{p}\right)^{2}}{2 m}-3.11 u_{p}, \\
b^{2}=\alpha_{1}^{2} \frac{\left(p_{\|}-2.24 e F / \omega\right)^{2}}{2 m} .
\end{gathered}
$$

For later use, it is convenient to rewrite Eq. (73) as 


$$
4 a c-b^{2}=4 a\left\{\xi_{1}\left[a I /\left(2 I_{\mathrm{at}}\right)\right]^{1 / 3} E_{\mathrm{at}}-\Delta\left|E_{0}\right|\right\} .
$$

This transcendental equation cannot be solved analytically with respect to $p_{n} \equiv p_{c}(\theta)$ for a fixed ejection angle $\theta$. We thus treat it approximately, taking into account that both $\gamma$ and $\hbar \omega /\left|E_{0}\right|$ are small in the tunneling limit. We express the cutoff momentum $p_{c}(\theta)$ as $p_{c}(\theta)=p_{c}^{(0)}(\theta)+\Delta p_{c}(\theta)$, where $p_{c}^{(0)}(\theta)$ satisfies the equation

$$
f\left(p_{c}^{(0)}(\theta), \theta\right) \equiv 4 a c-b^{2}=0 .
$$

We now expand the left-hand side (lhs) of Eq. (74) in $p$ about the point $p=p_{c}^{(0)}(\theta)$, retaining only the linear term, and consider the rhs of Eq. (74) as a perturbation, evaluating it at the point $p_{c}(\theta)=p_{c}^{(0)}(\theta)$. We thus obtain the following result for $\Delta p_{c}(\theta)$ :

$$
\Delta p_{c}(\theta)=\left.\frac{4 a\left\{\xi_{1}\left[a I /\left(2 I_{\mathrm{at}}\right)\right]^{1 / 3} E_{\mathrm{at}}-\Delta\left|E_{0}\right|\right\}}{d f(p, \theta) / d p}\right|_{p=p_{c}^{(0)}(\theta)} .
$$

Consider first the position of the cutoff at zero ejection angle, $\theta=0$. In this case the solution of Eq. (75) is

$$
p_{c}^{(0)} \equiv p_{c}^{(0)}(0)=2.24 \frac{e F}{\omega},
$$

which corresponds to a photoelectron energy equal to the classical cutoff energy $E_{c}^{\text {cl }}$ in Eq. (70). Substituting Eq. (77) into Eq. (76), we obtain the first quantum correction (of order $\gamma$ ) to $p_{c}^{(0)}$ for $\theta=0$ :

$$
\Delta p_{c}=\gamma \sqrt{2 m\left|E_{0}\right|}\left[0.12-0.54\left(\frac{I}{2 I_{\mathrm{at}}}\right)^{1 / 3} \frac{E_{\mathrm{at}}}{\left|E_{0}\right|}\right] .
$$

Combining results (77) and (78), the cutoff position for $\theta$ $=0$ is given by

$$
E_{c}=E_{c}^{\mathrm{cl}}+0.54\left|E_{0}\right|-2.42\left[I /\left(2 I_{\mathrm{at}}\right)\right]^{1 / 3} E_{\mathrm{at}} .
$$

If one omits the intensity-dependent correction in square brackets, then Eq. (79) reduces to the result of Ref. [29] in Eq. (71). The intensity-dependent correction in Eq. (79) describes the quantum spreading of the returning electron wave packet and governs the sign of the total quantum correction to the classical $10 u_{p}$ law. The intensity $I_{\text {cr }}$ at which the two corrections in Eq. (79) approximately compensate for each other is $I_{\mathrm{cr}} \approx 2.2 \times 10^{-2}\left(\left|E_{0}\right| / E_{\mathrm{at}}\right)^{3} I_{\mathrm{at}}$. For $I<I_{\mathrm{cr}}$, the extent of the ATI or ATD plateau is larger than $10 u_{p}$, while for $I>I_{\mathrm{cr}}$ the position of the cutoff lies below $10 u_{p}$. For $\mathrm{H}^{-}$and $\mathrm{F}^{-}, I_{\text {cr }}$ corresponds to intensities of about $1.6 \times 10^{10}$ and 1.5 $\times 10^{12} \mathrm{~W} / \mathrm{cm}^{2}$, respectively. This approximate compensation explains why classical result (70) is in better agreement with our more accurate estimate (79) and with the exact numerical results in Figs. 1 and 2 for $\theta=0$ as compared to cutoff law (71). (For rare-gas atoms, we estimate that this compensation occurs in the range $10^{14}-10^{15} \mathrm{~W} / \mathrm{cm}^{2}$.) In Fig. 3 we compare the cutoff position given by Eq. (79) with that obtained from the numerical solution of transcendental equation (73). One sees that analytical estimate (79) reproduces very well the exact results, while Eq. (71) significantly overestimates the cutoff energy.

In a way similar to that for the case $\theta=0$, Eqs. (74)-(76) allow one to estimate the position of the cutoff for nonzero

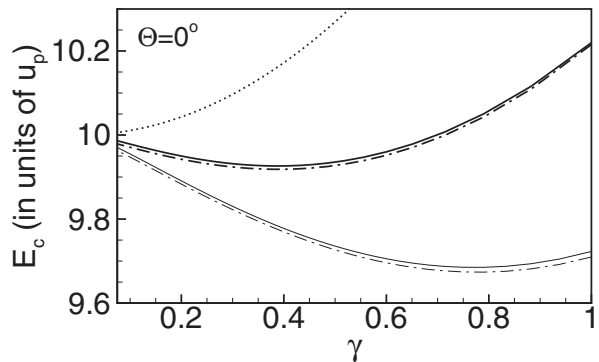

FIG. 3. Cutoff energy vs the Keldysh parameter $\gamma$ for $\theta=0$ and two frequencies, $\omega=0.075\left|E_{0}\right|$ (thick lines) and $\omega=0.15\left|E_{0}\right|$ (thin lines). Solid lines, exact results [cf. Eq. (73)]; dot-dashed lines, results of Eq. (79); dotted line, results of Ref. [29].

ejection angles, assuming that the angle $\theta$ (in radians) is small. The derivation is straightforward, but lengthy. Hence we present here only the final result:

$$
\begin{aligned}
E_{c}(\theta)= & E_{c}^{\mathrm{cl}}(\theta)+\left(0.54-0.21 \theta^{2}\right)\left|E_{0}\right| \\
& -\left(2.42-0.96 \theta^{2}\right)\left[I /\left(2 I_{\mathrm{at}}\right)\right]^{1 / 3} E_{\mathrm{at}},
\end{aligned}
$$

where $E_{c}^{\mathrm{cl}}(\theta)$ is given by Eq. (72). In Fig. 4 we compare results of analytic formula (80) with those obtained from the exact solution of Eq. (73). One sees that Eq. (80) describes quite well the $\theta$ dependence of the cutoff energy up to $\theta$ $\approx 35^{\circ}$, but significantly underestimates the cutoff energy as $\theta$ increases further. As for the results of Ref. [9] [i.e., Eq. (72)], one sees that they overestimate the cutoff energy for $\theta$ $\lesssim 32^{\circ}$ and underestimate the cutoff energy for $\theta \gtrsim 32^{\circ}$, approaching the results of Eq. (80).

\section{Interference oscillations in the energy and momentum distributions of ATD electrons}

Figures 1 and 2 show that the rates $\Gamma_{n}\left(\mathbf{p}_{n}\right)$ oscillate as functions of the photoelectron energy, $E_{n}=\mathbf{p}_{n}^{2} /(2 m)$. Qualitatively, this behavior is essentially independent of the ejection angle $\theta$. Near the high-energy end of the plateau, each of these oscillations involves more than ten photoelectron peaks. Such broad oscillation structures are known also in ATI spectra and have been found not only in theoretical results (obtained either by direct numerical solution of the TDSE-as in, e.g., Ref. [32] for atomic $\mathrm{H}-$ or by use of modified Keldysh theories [2,9]), but also in experimentally

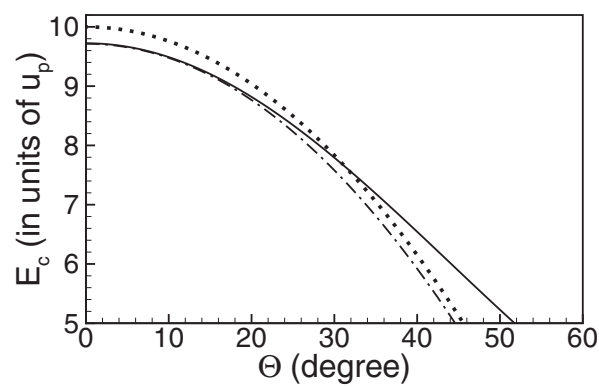

FIG. 4. Cutoff energy vs ejection angle $\theta$ for $\gamma=0.5$ and $\omega$ $=0.155\left|E_{0}\right|$. Solid line, exact results [cf. Eq. (73)]; dot-dashed line, results of Eq. (80); dotted line, results of Eq. (72). 
measured results (cf. Refs. [33,34]). According to Eq. (59), these oscillations originate from the oscillatory behavior of the propagation term $\mathcal{W}\left(\mathbf{p}_{n}\right)$, while the positions of their maxima and minima coincide with the positions $\left(\xi_{k}\right)$ of the maxima and minima of the Airy function $\operatorname{Ai}(\xi)$ for $k \geq 2$. [Recall that $\xi_{1}(=-1.019)$ determines the position of the plateau cutoff; cf. Sec. IV B.] For $k \geq 2$, the values of $\xi_{k}$ are approximated well (i.e., with an accuracy of a few percent) by equating to $\pi k / 2$ the argument of the sine function in the asymptotic form of $\mathrm{Ai}(-|\xi|)$ for large $|\xi|[31]$,

$$
\operatorname{Ai}(-|\xi|) \sim|\xi|^{-1 / 4} \sin \left(\frac{2}{3}|\xi|^{3 / 2}+\frac{\pi}{4}\right)
$$

The maxima (minima) of $\Gamma_{n}\left(\mathbf{p}_{n}\right)$, at the photoelectron energies $\left(E_{k}\right)_{\max (\min )}$, correspond to odd (even) $k$ in the relation

$$
\widetilde{\xi}=\xi_{k} \equiv-0.25[3 \pi(2 k-1)]^{2 / 3}, \quad k \geq 2,
$$

where $\widetilde{\xi}$ is given by Eq. (57). The energies $\left(E_{k}\right)_{\max (\min )}$ $\left(=\left[p_{k}(\theta)_{\max (\min )}\right]^{2} /(2 m)\right)$ are given by Eq. (74) upon substituting there $\xi_{1} \rightarrow \xi_{k}$ and solving it with respect to

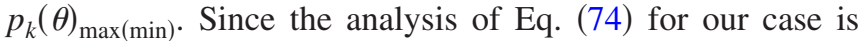
essentially the same as in Sec. IV B, we present only the final result:
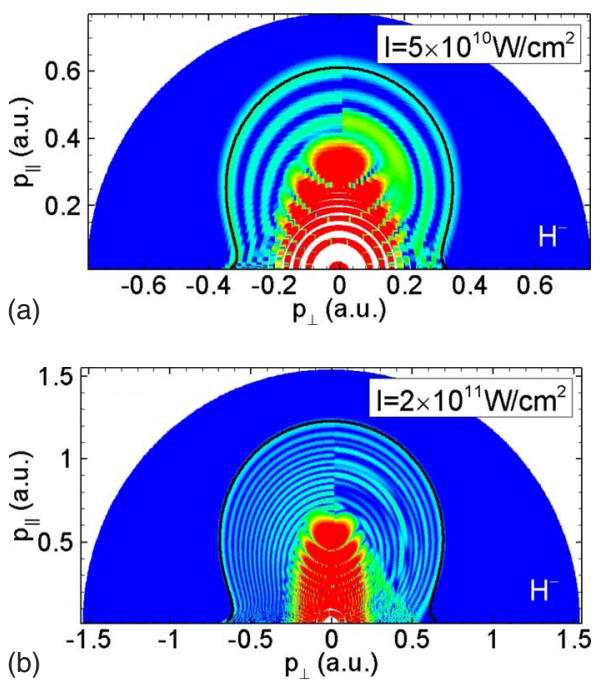

FIG. 5. (Color online) Electron momentum distributions following ATD of $\mathrm{H}^{-}$by a $\mathrm{CO}_{2}$ laser field $(\lambda=10.6 \mu \mathrm{m})$ plotted vs $p_{\|}$ $=p \cos \theta$ and $p_{\perp}=p \sin \theta$ (where $\theta$ is the angle between the polarization vector of the linearly polarized laser field and the momentum $\mathbf{p}$ of the detached electron). Results are shown for two intensities: (a) $I=5 \times 10^{10} \mathrm{~W} / \mathrm{cm}^{2} \quad(\gamma=0.848) ;$ (b) $I=2 \times 10^{11} \mathrm{~W} / \mathrm{cm}^{2}$ $(\gamma=0.424)$. The left half of each figure (i.e., $\left.p_{\perp}<0\right)$ shows our analytical results for $\Gamma_{n}\left(\mathbf{p}_{n}\right)$ [cf. Eq. (1)] obtained using Eqs. (37), (38), and (56). The right half of each figure (i.e., $p_{\perp}>0$ ) shows the corresponding exact TDER results. The narrow black line tracing the outer maximum of the momentum distribution in each figure marks the cutoff position as a function of angle according to Eq. (73); see the discussion in the text.
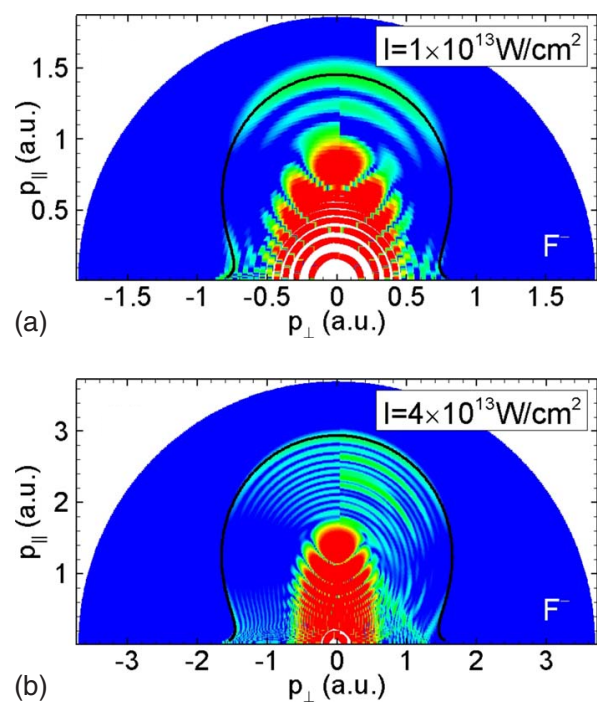

FIG. 6. (Color online) The same as in Fig. 5, but for ATD of $\mathrm{F}^{-}$ by a laser field of wavelength $\lambda=1.8 \mu \mathrm{m}$ and two intensities: (a) $I=1 \times 10^{13} \mathrm{~W} / \mathrm{cm}^{2} \quad(\gamma=0.75)$ and (b) $I=4 \times 10^{13} \mathrm{~W} / \mathrm{cm}^{2} \quad(\gamma$ $=0.375)$.

$$
\begin{aligned}
\left(E_{k}\right)_{\max (\min )}(\theta)= & E_{c}^{\mathrm{cl}}(\theta)+\left(0.54-0.21 \theta^{2}\right)\left|E_{0}\right| \\
& +\xi_{k}\left(2.37-0.93 \theta^{2}\right)\left[I /\left(2 I_{\mathrm{at}}\right)\right]^{1 / 3} E_{\mathrm{at}} .
\end{aligned}
$$

It is interesting to note that according to Eq. (83) the distance between maxima or minima in the ATI or ATD spectra for a given ejection angle depends only on the intensity:

$$
\frac{E_{k}-E_{m}}{E_{a t}}=\left(\xi_{k}-\xi_{m}\right)\left(2.37-0.93 \theta^{2}\right)\left[I /\left(2 I_{\mathrm{at}}\right)\right]^{1 / 3} .
$$

Thus, measurements of the distance between two maxima (or minima) in ATI or ATD spectra allow one to estimate the intensity of a laser field.

The interference oscillations are most spectacular in the momentum distribution of the ejected electrons. In Figs. 5 and 6 we present these distributions for ATD of $\mathrm{H}^{-}$and $\mathrm{F}^{-}$. In these calculations we used the total ATD amplitude in Eq. (37), including the Keldysh amplitude, so that the major differences between our analytic results for $\Gamma_{n}\left(\mathbf{p}_{n}\right)$ (shown in the left half of each figure) and the exact TDER results (shown in the right half of each figure) occur for energies from the onset to the middle part of the plateau.

The origin of the oscillation pattern in the ATI or ATD spectra becomes clear within the classical three-step scenario. As follows from the classical equation for the electron energy $E$ after rescattering [28], this energy depends on the initial time $\tau_{0}$ (corresponding to the ionization event), $\tau_{0}$ $\approx \widetilde{\tau}_{0}=-2.881$, and the moment $\tau$, at which the electron comes back and rescatters. For $E<10 u_{p}$, there are two closed classical trajectories ("long" and "short"), which correspond to the same final energy $E$, but have different return times $\tau$, denoted by $\tau_{+}$and $\tau_{-}$. As discussed in Ref. [9], the oscillation pattern in the ATI rates results from interference between these two trajectories. In our quantum analysis, the times $\tau_{+}$and $\tau_{-}$are given by Eq. (54). Evaluating the integral 
in Eq. (39) by the saddle-point method, it can be shown that the rescattering amplitude is (in scaled units)

$$
\tilde{\mathcal{A}}_{n}^{(l)}\left(\mathbf{p}_{n}\right) \sim e^{i\left[\Phi\left(\tau_{-}\right)+\pi / 4\right]}-e^{i\left[\Phi\left(\tau_{+}\right)-\pi / 4\right]} .
$$

Substituting $\tau_{ \pm}$into Eq. (48) for $\Phi(\tau)$, we obtain

$$
\Phi\left(\tau_{ \pm}\right)-\Phi_{0}=\mp \frac{\left(b^{2}-4 a c\right)^{3 / 2}}{12 F a^{2}}=\mp \frac{2}{3}|x|^{3 / 2},
$$

where $x$ is given by Eq. (57) upon omitting there the quantum correction $\Delta$. As a result, the amplitude $\tilde{\mathcal{A}}_{n}^{(l)}\left(\mathbf{p}_{n}\right)$ involves an oscillating factor, $\sin \left[(2 / 3)|x|^{3 / 2}+\pi / 4\right]$, which is the same one as in asymptotic form (81) of the Airy function for the case of $\Delta=0$. Therefore, the broad oscillations in the rates $\Gamma_{n}\left(\mathbf{p}_{n}\right)$ below the cutoff originate from interference of the contributions of the two saddle points $\tau_{ \pm}$or, in classical terms, from the interference of the short and long classical trajectories.

\section{Interference oscillations in the intensity and frequency dependences of the $\boldsymbol{n}$-photon ATD rate}

Interference oscillations, similar to those discussed in Sec. IV C, also take place in the intensity and frequency dependences of the ATD rate for a fixed number $n$ of absorbed photons. Indeed, even if for given intensity and laser wavelength the $n$-photon ATD peak occurs at the cutoff (so that only one trajectory contributes to the corresponding ATD amplitude), with increasing $I$ or $\lambda$ the position of this peak moves to the below-cutoff part of the plateau, where both saddle points $\tau_{ \pm}$contribute to the propagation factor $\mathcal{W}\left(\mathbf{p}_{n}\right)$ in Eq. (63) and cause interference oscillations. Condition (82) gives a transcendental equation for the intensities or frequencies corresponding to the minima and maxima of these oscillations.

In Figs. 7 and 8 we present the dependence of the ATD rate for $\mathrm{H}^{-}$as a function of intensity and frequency for $n$ $=150$. One sees that analytic result (59) predicts quite smooth oscillations in both the intensity and frequency dependences of the ATD rate (cf. dot-dashed lines in Figs. 7 and 8), while the exact numerical results show that these smooth oscillations are strongly modified. These modifications are caused by threshold phenomena that significantly affect the ATD rates at intensities (or frequencies) corresponding to the closure of the lowest open ( $n_{0}$-photon) detachment channel with variation in $I$ (or $\omega$ ) [33,35-38], i.e., whenever

$$
\left|E_{0}\right|+u_{p}=n_{0} \hbar \omega \text {. }
$$

[In our derivation of $\widetilde{\mathcal{A}}_{n}^{(l)}\left(\mathbf{p}_{n}\right)$, such threshold phenomena were omitted owing to substitution (26).] It is interesting to note that the threshold phenomena in Figs. 7 and 8 become visible even for ATD peaks at the cutoff. This contrasts with the case of high-harmonic generation, where threshold phenomena become important only in the middle part of the plateau for harmonic rates [37,39]. This difference stems from the fact that the effects of the atomic potential are much more important for the description of the ATD or ATI plateau than for the plateau in high-harmonic spectra. (Indeed, even

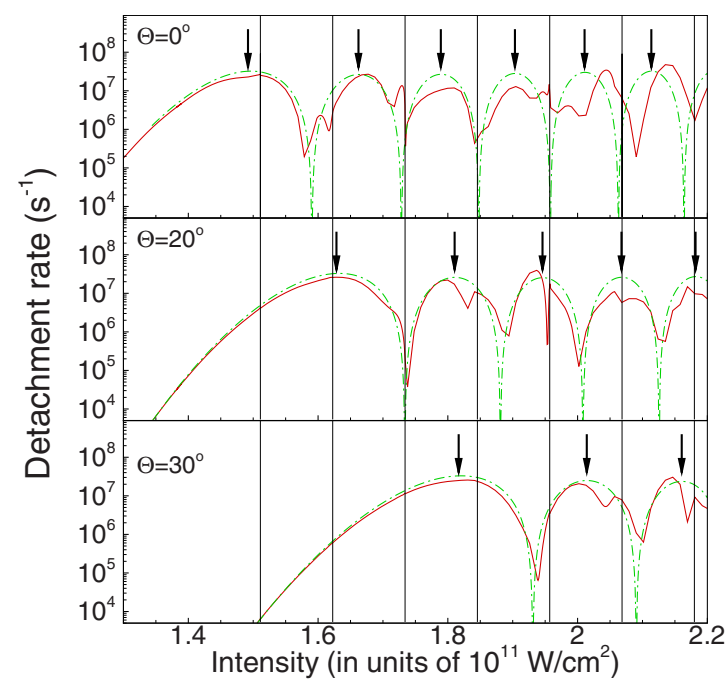

FIG. 7. (Color online) Intensity dependence of the ATD rate $\Gamma_{n}\left(\mathbf{p}_{n}\right)$ for $\mathrm{H}^{-}$for $n=150$ and the wavelength $\lambda=10.6 \mu \mathrm{m}$. Solid lines, exact numerical TDER results; dot-dashed lines, results of our analytic equation (59). Vertical solid lines mark the positions of the $n_{0}$-photon detachment thresholds [according to Eq. (87)] for 21 $\leq n_{0} \leq 27$. Arrows mark the positions of the maxima of the oscillation pattern in the intensity dependence of $\Gamma_{n}\left(\mathbf{p}_{n}\right)$ according to Eq. (82)

though laser-induced threshold phenomena are governed by both electron-atom and electron-laser interactions, for harmonic generation the atomic potential effects may be taken into account in lowest order, i.e., on the level of bound-state wave function [25], whereas the ATD or ATI plateau originates from higher order, rescattering effects.)

\section{E. Generalization to ATI of neutral atoms}

Our analytic results for ATD amplitudes and rates may be generalized to give an acceptable description of the high-

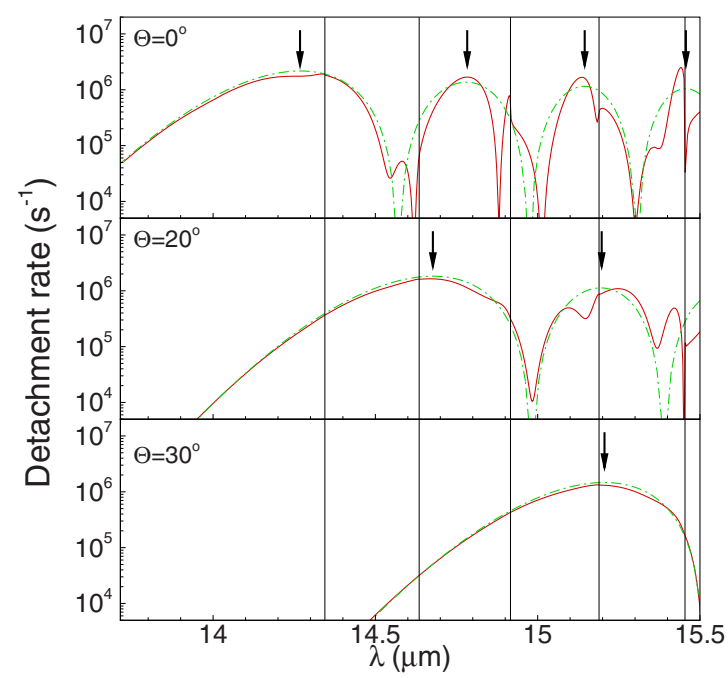

FIG. 8. (Color online) The same as in Fig. 7, but for the wavelength dependence of $\Gamma_{n}\left(\mathbf{p}_{n}\right)$ with $n=150$ for the intensity $I=6$ $\times 10^{10} \mathrm{~W} / \mathrm{cm}^{2}$. Vertical solid lines mark the positions of the $n_{0}$-photon detachment thresholds for $22 \leq n_{0} \leq 26$. 
energy part of the ATI spectrum for a neutral atom. This generalization is based on the following three considerations: first, analytic result (59) for the ATD rates represents, in fact, a "quantum replica" of the classical three-step scenario [28], which, as is commonly accepted, does not depend on the atomic species; second, as demonstrated in Secs. IV B-IV D, the results of our analytic formulas compare reasonably well with the exact numerical TDER results for ATD spectra for negative ions; and, third, each of the three terms in Eq. (59) has a transparent physical meaning and thus can be replaced by its corresponding atomic counterpart. In the following paragraphs, we discuss the atomic counterparts for each of the three terms in Eq. (59):

(i) The "Coulomb-modified" ionization factor $\mathcal{I}^{(a)}(F, \omega)$ is given by Eq. (61) in which $\Gamma_{\text {st }}(F)$ [cf. Eq. (62)] is replaced by the tunneling formula for ionization of an atomic electron by a static field, $\Gamma_{\mathrm{st}}^{(a)}(F)$. We parametrize the energy $E_{a}$ of an active atomic electron as $E_{a}=-\hbar^{2} \kappa_{a}^{2} /(2 m)$. As is well known $[27,40], \Gamma_{\mathrm{st}}^{(a)}(F)$ differs from $\Gamma_{\mathrm{st}}(F)$, first, by the "Coulomb factor" $\quad\left(4 F_{a} / F\right)^{2 \nu}, \quad$ where $\quad F_{a}=\sqrt{2 m\left|E_{a}\right|^{3}} /(e \hbar), \quad \nu$ $=Z \sqrt{E_{\text {at }} /\left(2\left|E_{a}\right|\right)}=Z /\left(\kappa_{a} a_{0}\right)$ is an effective principal quantum number, and $Z e$ is the charge of the remaining atomic core (i.e., $Z=0$ for negative ions and $Z=1$ for neutral atoms). Second, the asymptotic behavior (for $\kappa_{a} r \gg 1$ ) of the bound-state wave function for a Coulomb-type potential,

$$
\left.\psi_{\kappa_{a} l m}(\mathbf{r})\right|_{r \gg \kappa_{a}^{-1}}=C_{\kappa_{a}} l^{\nu-1} e^{-\kappa_{a} r} Y_{l, m}(\hat{\mathbf{r}}),
$$

differs in form from that in Eq. (4) for a short-range potential. Thus the term $C_{k l}^{2}$ in Eq. (62) should be replaced by $C_{\kappa_{l} l}^{2} \kappa_{a}^{-2 \nu}$. As a result, the ionization factor $\mathcal{I}(F, \omega)$ in Eq. (59) for the case of ATI is given by Eq. (61), where $\Gamma_{\mathrm{st}}(F)$ is replaced by

$$
\Gamma_{\mathrm{st}}^{(a)}(F)=\frac{\hbar}{2 m}(2 l+1) C_{\kappa_{a} l}^{2}\left(\frac{4 F_{a}}{\kappa_{a} F}\right)^{2 \nu-1} e^{-4 F_{a^{\prime}}(3 F)} .
$$

(ii) If we neglect the quantum correction $\left(\Delta\left|E_{0}\right|\right.$ $\left.=0.3\left|E_{0}\right|\right)$ in the argument $\xi$ of the Airy function, the propagation factor $\mathcal{W}\left(\mathbf{p}_{n}\right)$ in Eq. (63) does not involve any atomic parameters (since it describes free-electron motion in a laser field). Thus, its generalization to the case of ATI involves simply the replacement $\Delta\left|E_{0}\right| \rightarrow \Delta\left|E_{a}\right|$ in Eq. (64).

(iii) The cross section for elastic electron scattering from a short-range potential $\left(\sigma^{(l)}\right)$ in Eq. (59) should simply be replaced by that from a singly charged positive atomic ion, i.e., $\sigma^{(l)} \rightarrow \sigma_{a}$. In particular, for ATI of atomic hydrogen $\sigma_{a}$ is given by the Rutherford formula:

$$
\sigma(\mathbf{p})=\frac{m^{2} e^{4}}{p^{4}}\left(1+\frac{\mathbf{e}_{z} \cdot \mathbf{p}}{p}\right)^{-2}, \quad \mathbf{p}=\mathbf{p}_{n}-\Delta \mathbf{p} .
$$

Note, that in the shifted (in $\Delta \mathbf{p}$ ) reference frame, Eq. (90) reduces to usual Rutherford formula for elastic backscattering. This is a key point in Refs. $[15,16]$ that is found to be necessary to describe the "backrescattered ridges" in ATI angular distributions.

Upon making the replacements indicated above, our analytic formula in Eq. (59) for the ATD rate for a negative ion takes the following form for the ATI rate for a neutral atom:

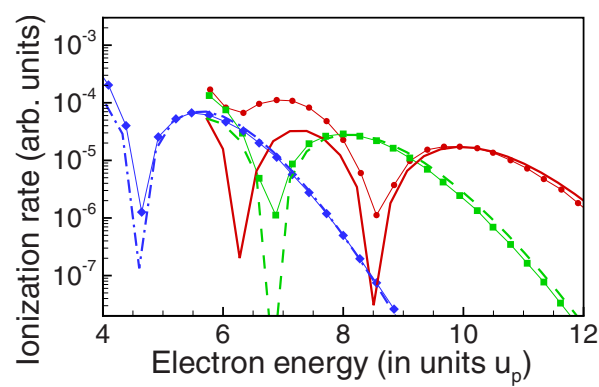

FIG. 9. (Color online) ATI spectrum of atomic hydrogen for a laser intensity $I=2 \times 10^{14} \mathrm{~W} / \mathrm{cm}^{2}$ and $\hbar \omega=2 \mathrm{eV}\left(u_{p}=7.18 \mathrm{eV}, \gamma\right.$ $=0.97$ ) for three electron ejection angles $\theta$ (with respect to the direction of linear laser polarization). Thin solid lines with symbols: TDSE results of Ref. [32] (circles, $\theta=0^{\circ}$; squares, $\theta=30^{\circ}$; diamonds, $\theta=50^{\circ}$ ). Thick lines without symbols: Coulomb-modified TDER results according to Eq. (91) (solid line, $\theta=0^{\circ}$; dashed line, $\theta=30^{\circ}$; dot-dashed line, $\theta=50^{\circ}$ ). The results obtained using Eq. (91) are shifted to higher energy by $0.4 u_{p}(=2.87 \mathrm{eV})$.

$$
\Gamma_{n}\left(\mathbf{p}_{n}\right)=\mathcal{I}^{(a)}(F, \omega) \mathcal{W}\left(\mathbf{p}_{n}\right) \sigma_{a}\left(\mathbf{p}_{n}-\Delta \mathbf{p}\right),
$$

where

$$
\mathcal{I}^{(a)}(F, \omega)=C_{\kappa_{a} l}^{2} \frac{2 l+1}{2 \pi \kappa_{a}} \gamma^{2}\left(\frac{4 F_{a}}{\kappa_{a} F}\right)^{2 \nu-1} e^{-4 F_{a} /(3 F)} .
$$

Note that in the Coulomb approximation [41], the factor $C_{\kappa_{a} l}^{2}$ for the active electron of a neutral atom is

$$
C_{\kappa_{a} l}^{2}=\frac{\kappa_{a}\left(2 \kappa_{a}\right)^{2 \nu}}{\nu \Gamma(\nu-l) \Gamma(\nu+l+1)},
$$

where $\Gamma(x)$ is the gamma function.

In Fig. 9 we compare results of our Coulomb-modified analytic result in Eq. (91) (for differential ATI rates along the high-energy part of the ATI plateau) with the benchmark results of Ref. [32] for ATI of the hydrogen atom obtained by numerical solution of the TDSE. Our results show that for all ejection angles $\theta$, the cutoff positions of the ATI spectra obtained in our calculations are shifted to lower energy as compared to those of Ref. [32] by the same constant energy $\left(\approx 0.4 u_{p}\right)$. As shown in Fig. 9, after shifting our results by this energy, the agreement of our results for the ATI spectra with those of Ref. [32] may be considered as perfect taking into account the approximate character of our results. (Note also that our results are formally valid in the tunneling limit, i.e., for $\gamma \ll 1$ and $\hbar \omega /\left|E_{a}\right| \ll 1$, while for the data in Fig. 9 these parameters are $\gamma=0.97 \approx 1$ and $\hbar \omega /\left|E_{a}\right|=0.15$.) The significant difference between the numerical TDSE results and those obtained using our Eq. (91) (and making the constant energy shift discussed above) at the minima of the oscillation patterns may be caused, in part, by the finite duration of the laser pulse ( $\approx 25 \mathrm{fs}$ ) used in Ref. [32]. Finally, we note that the shift in ATI spectra in Fig. 9 is somewhat puzzling. We suspect that determining its origin will require a more detailed comparison with TDSE results over a wide interval of laser intensities and frequencies in the region $\gamma<1$. 


\section{SUMMARY AND CONCLUSIONS}

To summarize the results of this paper, we note first that our analysis is based on our essentially exact TDER theory $[13,23]$. This theory combines the well-known effective range theory [22] for a single weakly bound electron in a short-range potential with the well-known quasistationary quasienergy state (or Floquet) theory [20] to describe the electron's interaction with a monochromatic laser field. In the tunneling limit and for electron energies near the cutoff of the ATD plateau, we have approximated the TDER formulation to obtain closed-form analytic formulas for ATD rates.

The closed-form analytic result for the ATD rate in Eq. (59) is remarkable in that it is the product of three factors, each one of which corresponds to one of the three steps of the well-known rescattering scenario $[42,43]$. Moreover, if the factor corresponding to elastic electron scattering from the atomic core is removed, the remaining part of this formula gives an analytic expression for the electron wave packet introduced phenomenologically in recent analyses of ATI processes [15-19]. This electron wave packet occurs similarly in ATD processes. Moreover, since it corresponds to free-electron motion in the laser field, it is not significantly dependent on the atomic species from which the active electron originates since this dependence stems only from the ionization factor. Most importantly, the numerical predictions of ATD rates provided by Eq. (59) agree very well with the exact numerical results of our TDER theory, as demonstrated in the many detailed comparisons presented in Secs. IV B-IV D above. The only disagreements relate to threshold effects arising from multichannel interactions at particular values of laser intensity and frequency corresponding to the closure of a multiphoton detachment channel. Such multichannel interactions are ignored in our quasiclassical derivation of the analytic formula for the ATD rate and hence cannot be expected to appear in the predictions of the formula.

Owing to the fact that each of the three factors of the closed-form analytic formula for the ATD rate in Eq. (59) has a clearly defined physical interpretation, we have been able to generalize it quite straightforwardly to be applicable for ATI rates. That is, we simply introduce appropriate modifications originating from the fact that in neutral atoms the active electron moves in the Coulomb field of an ionic core. These modifications apply mainly to the factor corresponding to elastic electron scattering from the atomic core. Other modifications arise from the different asymptotic form of the initial bound-state wave function of the active electron. The resulting closed-form analytic formula for the ATI rate is given in Eq. (91). As shown in Fig. 9, the results of this analytic formula (when shifted in energy by $2.87 \mathrm{eV}$ ) agree essentially exactly in shape with the benchmark results of Ref. [32] on the ATI spectrum of atomic H, obtained by direct numerical solution of the TDSE. The reasons why a shift in energy is necessary to have nearly a perfect match of the predictions of our analytic formula and the results of solving the TDSE are at present unclear to us. Moreover, elucidation of this issue probably requires further comparisons over a much wider range of laser parameters.

We conclude by noting that our closed-form analytic formulas for both ATD and ATI rates [in Eqs. (59) and (91), respectively] give one analytical insight into how these rates may be controlled by varying the laser field parameters or by varying the atomic species. Note also that our results provide more accurate analytic formulas than obtained previously by others for ATD and ATI plateau cutoff energies as a function of angle, as discussed in Sec. IV B. Finally, in presenting an analytic expression for the phenomenological electron wave packet that has been introduced in a number of analyses of ATI processes [15-19], we provide a means of obtaining directly the elastic-electron-scattering cross sections from investigations of ATI and ATD processes.

\section{ACKNOWLEDGMENTS}

This work was supported in part by RFBR Grant No. 07-02-00574 and by NSF Grant No. PHY-0601196.

\section{APPENDIX A: DERIVATION OF EQ. (5)}

We use the spectral expansion of the Green's function $G\left(\mathbf{r}, t ; \mathbf{r}^{\prime}, t^{\prime}\right)$ in terms of Volkov wave functions $\Psi_{\mathbf{p}}(\mathbf{r}, t)$,

$$
G\left(\mathbf{r}, t ; \mathbf{r}^{\prime}, t^{\prime}\right)=\frac{\Theta\left(t-t^{\prime}\right)}{i \hbar} \int \Psi_{\mathbf{p}}(\mathbf{r}, t) \Psi_{\mathbf{p}}^{*}\left(\mathbf{r}^{\prime}, t^{\prime}\right) d \mathbf{p},
$$

where $\Theta\left(t-t^{\prime}\right)$ is the Heaviside (step) function. The Volkov wave functions have the following form:

$$
\begin{gathered}
\Psi_{\mathbf{p}}(\mathbf{r}, t)=e^{-(i / h) \epsilon_{p} t} \Phi_{\mathbf{p}}(\mathbf{r}, t), \\
\Phi_{\mathbf{p}}(\mathbf{r}, t)=(2 \pi \hbar)^{-3 / 2} \exp \left[i \mathcal{S}_{\mathbf{p}}(\mathbf{r}, t) / \hbar\right],
\end{gathered}
$$

where $\epsilon_{p}=p^{2} /(2 m)+u_{p}$ and

$$
\begin{aligned}
\mathcal{S}_{\mathbf{p}}(\mathbf{r}, t)= & \frac{e \mathbf{r} \cdot \dot{\mathbf{F}}(t)}{\omega^{2}}+\mathbf{p} \cdot\left(\mathbf{r}-\frac{e \mathbf{F}(t)}{m \omega^{2}}\right) \\
& -\int^{t}\left(\frac{e^{2}[\dot{\mathbf{F}}(t)]^{2}}{2 m \omega^{4}}-u_{p}\right) d t, \quad \mathbf{F}(t)=\mathbf{F} \cos \omega t .
\end{aligned}
$$

Substituting Eq. (A1) into Eq. (3) for $\chi\left(\mathbf{r}, \mathbf{r}^{\prime}, t\right)$, we obtain

$$
\begin{aligned}
\chi\left(\mathbf{r}, \mathbf{r}^{\prime}, t\right)= & \frac{i 2 \pi \hbar}{m} \int d \mathbf{p} \int_{-\infty}^{t} d t^{\prime} f\left(t^{\prime}\right) \\
& \times e^{(i / \hbar)\left(\epsilon-\epsilon_{p}\right)\left(t-t^{\prime}\right)} \Phi_{\mathbf{p}}(\mathbf{r}, t) \Phi_{\mathbf{p}}^{*}\left(\mathbf{r}^{\prime}, t^{\prime}\right) .
\end{aligned}
$$

The function $\Phi_{\mathbf{p}}^{*}\left(\mathbf{r}^{\prime}, t^{\prime}\right) f\left(t^{\prime}\right)$ is periodic in the time $t^{\prime}$, so that we expand it in a Fourier series and perform the integration over $t^{\prime}$ in Eq. (A4) to obtain (cf. Ref. [23]):

$$
\begin{aligned}
\chi\left(\mathbf{r}, \mathbf{r}^{\prime} ; t\right)= & -\frac{1}{4 \pi^{2} m \hbar} \sum_{s} \frac{1}{T} \int_{0}^{T} d t^{\prime} f\left(t^{\prime}\right) \\
& \times \int d \mathbf{p} \frac{e^{i\left[\mathcal{S}_{\mathbf{p}}(\mathbf{r}, t)-\mathcal{S}_{\mathbf{p}}\left(\mathbf{r}^{\prime}, t^{\prime}\right)\right] / \hbar-i s \omega\left(t-t^{\prime}\right)}}{\epsilon-\epsilon_{p}+s \hbar \omega+i 0} .
\end{aligned}
$$

The integration over the momentum $\mathbf{p}$ in Eq. (A5) may be performed analytically and yields Eq. (5) above.

\section{APPENDIX B: QUASICLASSICAL LIMIT OF THE NONDIAGONAL MATRIX ELEMENTS $M_{k, k^{\prime}}^{(l)}(\epsilon)$}

One-dimensional integral representation (15) for $M_{k, k^{\prime}}^{(l)}(\epsilon)$ is the most appropriate one for numerical calculations. How- 
ever, for approximate analytic evaluations of $M_{k, k^{\prime}}^{(l)}(\epsilon)$ in the quasiclassical limit $\left(\hbar \omega \ll\left|E_{0}\right|\right)$ an alternative representation, derived in this appendix, is more convenient. In what follows, we use scaled units and consider the nondiagonal $(k$ $\neq 0)$ matrix elements $M_{k, 0}^{(l)}(\epsilon) \equiv M_{k, 0}^{(l)}$ [which are equivalent to $M_{k+m, m}^{(l)}(\epsilon-2 m \omega)$ in view of symmetry relation (17)].

Consider first the case of a bound state of $s$ symmetry. According to the TDER theory [13], the $s$-wave part of the QQES wave function $\Phi_{\epsilon}(\mathbf{r}, t)$ satisfies, on one hand, the following boundary condition for $r \leqq r_{c}$ :

$$
\Phi_{\epsilon}(\mathbf{r}, t)=\frac{C_{\kappa l}}{\sqrt{4 \pi}} \sum_{k} e^{-2 i k \omega t}\left\{\frac{1}{r}+\left[-\frac{1}{a_{0}}+\frac{r_{0}}{2}(\epsilon+2 k \omega)\right]\right\} f_{2 k},
$$

where $f_{2 k} \equiv f_{2 k}^{(l=0)}$ and $a_{0}$ and $r_{0}$ are the $s$-wave scattering length and effective range, respectively. On the other hand, for $r \gtrsim r_{c}$, the QQES wave function is given by Eq. (2),

$$
\begin{aligned}
\Phi_{\epsilon}(\mathbf{r}, t) & =-\sqrt{4 \pi} C_{\kappa l} \int_{-\infty}^{t} d t^{\prime} G\left(\mathbf{r}, t ; 0, t^{\prime}\right) f\left(t^{\prime}\right) e^{i \epsilon\left(t-t^{\prime}\right)} \\
& =\frac{C_{\kappa l}}{\sqrt{4 \pi}} \chi(\mathbf{r}, 0, t),
\end{aligned}
$$

where $\chi\left(\mathbf{r}, \mathbf{r}^{\prime}, t\right)$ is defined by Eq. (5). For small $r\left(r \sim r_{c}\right)$, the $s$-wave part of function (B2) has the form

$$
\begin{aligned}
\int \Phi_{\epsilon}(\mathbf{r}, t) d \Omega_{\mathbf{r}}= & \sqrt{4 \pi} C_{\kappa l} \sum_{k} e^{-2 i k \omega t}\left(\frac{f_{2 k}}{r}+\sum_{k^{\prime}}\left[i \sqrt{\epsilon+2 k \omega} \delta_{k, k^{\prime}}\right.\right. \\
& \left.\left.+M_{k, k^{\prime}}^{(0)}\right] f_{2 k^{\prime}}\right),
\end{aligned}
$$

where we have used the relation [cf. Eq. (13)]

$$
\left[-\frac{1}{a_{0}}+\frac{r_{0}}{2}(\epsilon+2 k \omega)\right] f_{2 k}=\sum_{k^{\prime}}\left(i \sqrt{\epsilon+2 k \omega} \delta_{k, k^{\prime}}+M_{k, k^{\prime}}\right) f_{2 k^{\prime}} .
$$

Upon substituting $f(t) \equiv 1$ (or, equivalently, $f_{2 k} \equiv \delta_{k 0}$ ) in Eqs. (B2) and (B3) [i.e., using the function $\widetilde{\Phi}_{\epsilon}(\mathbf{r}, t)$ $\left.=\left.\Phi_{\epsilon}(\mathbf{r}, t)\right|_{f(t)=1}\right]$, the matrix elements $M_{k, 0}^{(0)}$ with $k \neq 0$ are given by the $r$-dependent Fourier coefficients of the function $\int \widetilde{\Phi}_{\epsilon}(\mathbf{r}, t) d \Omega_{\mathbf{r}}$ upon substituting there $r \rightarrow 0$, i.e.,

$$
M_{k, 0}^{(0)}=\left(C_{\kappa l} \sqrt{4 \pi} T\right)^{-1} \lim _{r \rightarrow 0} \int_{0}^{T} \widetilde{\Phi}_{\epsilon}(\mathbf{r}, t) e^{2 i k \omega t} d \Omega_{\mathbf{r}} d t,
$$

which may be represented as

$$
M_{k, 0}^{(0)}=-\frac{4 \pi}{T} \int_{0}^{T} d t \int_{0}^{\infty} d \tau G(0, t ; 0, t-\tau) e^{i \epsilon \tau+2 i k \omega t} .
$$

Using the explicit form (5) for $\chi\left(\mathbf{r}, \mathbf{r}^{\prime}, t\right)$ and substituting there $f(t)=1$, the matrix element $M_{k, 0}^{(0)}$ for $k \neq 0$ takes the form

$$
M_{k, 0}^{(0)}=\frac{\omega^{2}}{2 F T^{2}} \sum_{s} \iint \frac{e^{i\left[S\left(-\tilde{\mathbf{K}}_{s}, t^{\prime}\right)-S\left(-\tilde{\mathbf{K}}_{s}, t\right)+2 k \omega t\right]}}{\left|\cos \omega t-\cos \omega t^{\prime}\right|} d t d t^{\prime},
$$

where both integrals are taken over the period $T=2 \pi / \omega$,

$$
\begin{gathered}
\widetilde{\mathbf{K}}_{s} \equiv \widetilde{\mathbf{K}}_{s}\left(t, t^{\prime}\right)=\mathbf{K}_{s} \frac{\cos \omega t-\cos \omega t^{\prime}}{\left|\cos \omega t-\cos \omega t^{\prime}\right|}, \\
\mathbf{K}_{s}=\mathbf{e}_{z} k_{s}, \quad k_{s}=\sqrt{\epsilon+s \omega-u_{p}}, \\
S\left(-\widetilde{\mathbf{K}}_{s}, t\right)=\int^{t}\left[\left(\widetilde{\mathbf{K}}_{s}\left(t, t^{\prime}\right)+\frac{\mathbf{F}}{\omega} \sin \omega \tau\right)^{2}-\epsilon\right] d \tau .
\end{gathered}
$$

For the case of a bound state of $p$ symmetry, the derivation is more cumbersome since the small- $r$ boundary condition, similar to that in Eq. (B1), involves additional terms, $\sim r^{-2}$ and $\sim r[13,23]$, and differentiation of the function $\chi\left(\mathbf{r}, \mathbf{r}^{\prime}, t\right)$ with respect to $z^{\prime}$ is necessary [cf. Eq. (2)]. Thus we omit these lengthy calculations and present only the final result for the matrix element $M_{k, 0}^{(1)}(\epsilon)$ :

$$
\begin{aligned}
M_{k, 0}^{(1)}(\epsilon)= & \frac{3 \omega^{2}}{2 F T^{2}} \sum_{s} \iint \frac{e^{i\left[S\left(-\tilde{\mathbf{K}}_{s}, t^{\prime}\right)-S\left(-\tilde{\mathbf{K}}_{s}, t\right)+2 k \omega t\right]}}{\left|\cos \omega t-\cos \omega t^{\prime}\right|} \\
& \times\left(\widetilde{\mathbf{K}}_{s}+\frac{\mathbf{F}}{\omega} \sin \omega t\right) \cdot\left(\widetilde{\mathbf{K}}_{s}+\frac{\mathbf{F}}{\omega} \sin \omega t^{\prime}\right) d t d t^{\prime} .
\end{aligned}
$$

Carrying out the integrations over $t^{\prime}$ in Eqs. (B7) and (B8) by the saddle-point method (in a way similar to that in Appendix B of Ref. [23]), the two results may be given in a unified form applicable for both $s$ and $p$ states. This form is given by Eq. (21) of the main text.

\section{APPENDIX C: ALTERNATIVE DERIVATION FOR THE IONIZATION FACTOR IN EQ. (60)}

In this appendix we show how Eq. (60) follows from the quasiclassical (saddle-point) treatment of Keldysh amplitude (38) in the limit $p_{n} \rightarrow 0$. For simplicity, all formulas are given in scaled units (cf. Sec. II).

The saddle points for the integral in Eq. (38) are given by the equation

$$
\left(\mathbf{p}_{n}-\frac{\mathbf{F}}{\omega} \sin \tau\right)^{2}=-1,
$$

which has two (complex-conjugated) pairs of roots: two of these roots have positive imaginary parts, while those of the other two are negative. Only the roots with positive imaginary parts $\left(\tau_{1}\right.$ and $\left.\tau_{2}\right)$ contribute to the Keldysh amplitude in the limit $\gamma \rightarrow 0$ [40]. Moreover, if $\tau_{1}$ is the solution of Eq. (C1) with the smallest real part, then the second solution $\left(\tau_{2}\right)$ is $\tau_{2}=\pi-\tau_{1}^{*}$. In the lowest order in $p_{n}$, the result for $\tau_{1}$ is [40]

$$
\tau_{1}=i \operatorname{arcsinh}(\gamma)+\gamma p_{\|} / \sqrt{1+\gamma^{2}} .
$$

Using the relation between $\tau_{1}$ and $\tau_{2}$, it can be shown that the second derivative of $S\left(\mathbf{p}_{n}, \tau\right)$ is the same for both saddle points in the limit $p_{n} \rightarrow 0$ : 


$$
S^{\prime \prime}\left(\mathbf{p}_{n}, \tau_{1}\right)=S^{\prime \prime}\left(\mathbf{p}_{n}, \tau_{2}\right)=-\frac{2 i F}{\omega^{2}} \sqrt{1+\gamma^{2}} \approx-\frac{2 i F}{\omega^{2}} .
$$

Expansion of $S\left(\mathbf{p}_{n}, \tau\right)$ at $\tau=\tau_{1}$ and $\tau_{2}$ up to terms $\sim p_{n}^{2}$ has the form

$$
\begin{gathered}
S\left(\mathbf{p}_{n}, \tau_{1}\right)=i g\left(p_{n}, \theta\right)+\frac{2 F p_{\|}}{\omega^{2}}, \\
S\left(\mathbf{p}_{n}, \tau_{2}\right)=i g\left(p_{n}, \theta\right)-\frac{2 F p_{\|}}{\omega^{2}}+\pi n,
\end{gathered}
$$

where

$$
g\left(p_{n}, \theta\right)=\frac{2}{3 F}\left[1+\frac{1}{2}\left(3 \sin ^{2} \theta+\gamma^{2}\right) p_{n}^{2}\right] .
$$

Evaluating the integral in Eq. (38) by the saddle-point method using Eqs. (C2)-(C4) and then taking into account the $p_{n}$-dependent terms only in the exponential, the square of the modulus of $\mathcal{A}_{n}^{(K)}\left(\mathbf{p}_{n}\right)$ takes the form

$$
\left|\mathcal{A}_{n}^{(K)}\left(\mathbf{p}_{n}\right)\right|^{2}=\frac{(2 l+1) C_{\kappa l}^{2} \omega^{2}}{8 \pi^{2} F} e^{-2 g\left(p_{n}, \theta\right)} .
$$

Neglecting the term $\sim p_{n}^{2}$ in Eq. (C5) for $g\left(p_{n}, \theta\right)$, Eq. (C6) yields the ionization factor $\mathcal{I}(F, \omega)$ in Eq. (60) after converting from scaled to absolute units and multiplying by $2 \pi$.

The factor $\mathcal{I}(F, \omega)$ may be connected also with the total detachment rate in the Keldysh approximation, $\Gamma^{(\mathrm{KA})}$, which is given by [cf. Eq. (1)]

$$
\begin{aligned}
\Gamma^{(\mathrm{KA})}= & \sum_{n} 2 p_{n} \int\left|\mathcal{A}_{n}^{(K)}\left(\mathbf{p}_{n}\right)\right|^{2} d \Omega_{\hat{\mathbf{p}}_{n}} \\
= & \frac{2 l+1}{\pi} C_{\kappa l}^{2} \gamma e^{-4 /(3 F)} \\
& \times \int_{0}^{\infty} d p \int_{0}^{\pi} d \theta p^{2} \sin \theta e^{-2 p^{2}\left(3 \sin ^{2} \theta+\gamma^{2}\right) /(3 F)} \\
= & \sqrt{\frac{3}{32 \pi}} \frac{(2 l+1) C_{\kappa l}^{2}}{1+\gamma^{2} / 3} F^{3 / 2} e^{-4 /(3 F)},
\end{aligned}
$$

where we have replaced the summation over $n$ by an integration over $p$ in a way similar to Eq. (26) (after which the integrations over $p$ and $\theta$ are performed analytically). Neglecting the term $\gamma^{2} / 3$ in the denominator, $\Gamma^{(\mathrm{KA})}$ in Eq. (C6) reduces to the well-known result for the total detachment rate in the quasistatic (low-frequency) limit [40]:

$$
\Gamma^{(\mathrm{KA})}=\sqrt{\frac{3 F}{2 \pi}} \Gamma_{\mathrm{st}}(F),
$$

where

$$
\Gamma_{\mathrm{st}}(F)=\frac{(2 l+1) C_{\kappa l}^{2}}{4} F e^{-4 /(3 F)}
$$

is the detachment rate of a weakly bound state $\psi_{\kappa l m}(\mathbf{r})$ having angular momentum $l$ and $m=0$ in a static electric field $\mathbf{F}$ [27].
[1] L. F. DiMauro and P. Agostini, Adv. At., Mol., Opt. Phys. 35, 79 (1995).

[2] W. Becker, F. Grabson, R. Kopold, D. B. Milošević, G. G. Paulus, and H. Walther, Adv. At., Mol., Opt. Phys. 48, 35 (2002).

[3] L. V. Keldysh, Zh. Eksp. Teor. Fiz. 47, 1945 (1964) [Sov. Phys. JETP 20, 1307 (1965)].

[4] M. Lewenstein, K. C. Kulander, K. J. Schafer, and P. H. Bucksbaum, Phys. Rev. A 51, 1495 (1995).

[5] A. Lohr, M. Kleber, R. Kopold, and W. Becker, Phys. Rev. A 55, R4003 (1997).

[6] D. B. Milošević and F. Ehlotzky, Phys. Rev. A 58, 3124 (1998).

[7] S. P. Goreslavskii and S. V. Popruzhenko, Phys. Lett. A 249, 477 (1998); Pis'ma Zh. Eksp. Teor. Fiz. 68, 858 (1998) [JETP Lett. 68, 902 (1998)].

[8] C. Figueira de Morisson Faria, H. Schomerus, and W. Becker, Phys. Rev. A 66, 043413 (2002).

[9] S. P. Goreslavskii and S. V. Popruzhenko, J. Phys. B 32, L531 (1999); Zh. Eksp. Teor. Fiz. 117, 895 (2000) [Sov. Phys. JETP 90, 778 (2000)].

[10] B. Borca, M. V. Frolov, N. L. Manakov, and A. F. Starace, Phys. Rev. Lett. 87, 133001 (2001).

[11] N. L. Manakov, M. V. Frolov, B. Borca, and A. F. Starace, J. Phys. B 36, R49 (2003).
[12] N. L. Manakov and A. G. Fainshtein, Zh. Eksp. Teor. Fiz. 79, 751 (1980) [Sov. Phys. JETP 52, 382 (1980)].

[13] M. V. Frolov, N. L. Manakov, E. A. Pronin, and A. F. Starace, Phys. Rev. Lett. 91, 053003 (2003).

[14] M. V. Frolov, N. L. Manakov, E. A. Pronin, and A. F. Starace, J. Phys. B 36, L419 (2003).

[15] Z. Chen, T. Morishita, A. T. Le, and C. D. Lin, Phys. Rev. A 76, 043402 (2007); T. Morishita, A. T. Le, Z. Chen, and C. D. Lin, New J. Phys. 10, 025011 (2008).

[16] T. Morishita, A. T. Le, Z. Chen, and C. D. Lin, Phys. Rev. Lett. 100, 013903 (2008).

[17] X. X. Zhou, Z. Chen, T. Morishita, A. T. Le, and C. D. Lin, Phys. Rev. A 77, 053410 (2008).

[18] M. Okunishi, T. Morishita, G. Prumper, K. Shimada, C. D. Lin, S. Watanabe, and K. Ueda, Phys. Rev. Lett. 100, 143001 (2008).

[19] D. Ray, B. Ulrich, I. Bocharova, C. Maharjan, P. Ranitovic, B. Gramkow, M. Magrakvelidze, S. De, I. V. Litvinyuk, A. T. Le, T. Morishita, C. D. Lin, G. G. Paulus, and C. L. Cocke, Phys. Rev. Lett. 100, 143002 (2008).

[20] N. L. Manakov, V. D. Ovsiannikov, and L. P. Rapoport, Phys. Rep. 141, 319 (1986).

[21] N. L. Manakov, M. V. Frolov, A. F. Starace, and I. I. Fabrikant, J. Phys. B 33, R141 (2000).

[22] L. D. Landau and E. M. Lifshitz, Quantum Mechanics, 4th ed. 
(Pergamon, Oxford, 1980)

[23] M. V. Frolov, N. L. Manakov, and A. F. Starace, Phys. Rev. A 78, 063418 (2008).

[24] A. I. Baz', Ya. B. Zel'dovich, and A. M. Perelomov, Scattering, Reactions, and Decays in Nonrelativistic Quantum Mechanics, 2nd ed. (Nauka, Moscow, 1971).

[25] M. V. Frolov, A. A. Khuskivadze, N. L. Manakov, and A. F. Starace, J. Phys. B 39, S283 (2006).

[26] N. Bleistein and R. Handelsman, Asymptotic Expansions of Integrals (Dover, New York, 1986); R. Wong, Asymptotic Approximations of Integrals (Academic, Boston, 1989).

[27] B. M. Smirnov and M. I. Chibisov, Zh. Eksp. Teor. Fiz. 49, 841 (1965) [Sov. Phys. JETP 22, 585 (1966)].

[28] G. G. Paulus, W. Becker, W. Nicklich, and H. Walther, J. Phys. B 27, L703 (1994).

[29] M. Busuladžić, A. Gazibegović-Busuladžić, and D. B. Milošević, Laser Phys. 16, 289 (2006).

[30] M. Lewenstein, Ph. Balcou, M. Yu. Ivanov, A. L'Huillier, and P. B. Corkum, Phys. Rev. A 49, 2117 (1994).

[31] Handbook of Mathematical Functions, edited by M. Abramowitz and I. A. Stegun (Dover, New York, 1965).

[32] E. Cormier and P. Lambropoulos, J. Phys. B 30, 77 (1997).

[33] G. G. Paulus, F. Grasbon, H. Walther, R. Kopold, and W.
Becker, Phys. Rev. A 64, 021401(R) (2001).

[34] C. Cornaggia, Phys. Rev. A 78, 041401(R) (2008).

[35] R. Kopold, W. Becker, M. Kleber, and G. G. Paulus, J. Phys. B 35, 217 (2002); S. V. Popruzhenko, Ph. A. Korneev, S. P. Goreslavski, and W. Becker, Phys. Rev. Lett. 89, 023001 (2002).; D. B. Milošević, E. Hasović, M. Busuladžić, A. Gazibegović-Busuladžić, and W. Becker, Phys. Rev. A 76, 053410 (2007).

[36] B. Borca, M. V. Frolov, N. L. Manakov, and A. F. Starace, Phys. Rev. Lett. 88, 193001 (2002).

[37] N. L. Manakov and M. V. Frolov, Pis'ma Zh. Eksp. Teor. Fiz. 83, 630 (2006) [JETP Lett. 83, 536 (2006)].

[38] K. Krajewska, I. I. Fabrikant, and A. F. Starace, Phys. Rev. A 74, 053407 (2006).

[39] B. Borca, A. F. Starace, A. V. Flegel, M. V. Frolov, and N. L. Manakov, Phys. Rev. A 65, 051402(R) (2002).

[40] A. M. Perelomov, V. S. Popov, and M. V. Terent'ev, Zh. Eksp. Teor. Fiz. 50, 1393 (1966) [Sov. Phys. JETP 23, 924 (1966)].

[41] D. Bates and A. Damgaard, Philos. Trans. R. Soc. London, Ser. A 242, 101 (1949).

[42] K. J. Schafer, B. Yang, L. F. DiMauro, and K. C. Kulander, Phys. Rev. Lett. 70, 1599 (1993).

[43] P. B. Corkum, Phys. Rev. Lett. 71, 1994 (1993). 